\title{
Role of CXCR2/CXCR2 ligands in vascular remodeling during bronchiolitis obliterans syndrome
}

\author{
John A. Belperio, ${ }^{1}$ Michael P. Keane, ${ }^{1}$ Marie D. Burdick, ${ }^{1}$ Brigitte Gomperts, ${ }^{2}$ Ying Ying Xue, ${ }^{1}$
} Kurt Hong, ${ }^{3}$ Javier Mestas, ${ }^{1}$ Abbas Ardehali, ${ }^{4}$ Borna Mehrad, ${ }^{5}$ Rajan Saggar, ${ }^{1}$ Joseph P. Lynch III, ${ }^{1}$ David J. Ross, ${ }^{1}$ and Robert M. Strieter ${ }^{1,2,6}$

1Department of Medicine, Division of Pulmonary and Critical Care Medicine, ${ }^{2}$ Department of Pediatrics, ${ }^{3}$ Department of Nutrition, and ${ }^{4}$ Department of Surgery, David Geffen School of Medicine at UCLA, Los Angeles, California, USA. ${ }^{5}$ Department of Medicine, Division of Pulmonary and Critical Care Medicine, The University of Texas Southwestern Medical Center, Dallas, Texas, USA. ${ }^{6}$ Department of Pathology and Laboratory Medicine, David Geffen School of Medicine at UCLA, Los Angeles, California, USA.

\begin{abstract}
Angiogenesis and vascular remodeling support fibroproliferative processes; however, no study has addressed the importance of angiogenesis during fibro-obliteration of the allograft airway during bronchiolitis obliterans syndrome (BOS) that occurs after lung transplantation. The ELR ${ }^{+}$CXC chemokines both mediate neutrophil recruitment and promote angiogenesis. Their shared endothelial cell receptor is the G-coupled protein receptor CXC chemokine receptor 2 (CXCR2). We found that elevated levels of multiple ELR ${ }^{+}$CXC chemokines correlated with the presence of BOS. Proof-of-concept studies using a murine model of BOS not only demonstrated an early neutrophil infiltration but also marked vascular remodeling in the tracheal allografts. In addition, tracheal allograft $\mathrm{ELR}^{+} \mathrm{CXC}$ chemokines were persistently expressed even in the absence of significant neutrophil infiltration and were temporally associated with vascular remodeling during fibro-obliteration of the tracheal allograft. Furthermore, in neutralizing studies, treatment with anti-CXCR2 Abs inhibited early neutrophil infiltration and later vascular remodeling, which resulted in the attenuation of murine BOS. A more profound attenuation of fibro-obliteration was seen when CXCR2 ${ }^{-/-}$mice received cyclosporin $\mathrm{A}$. This supports the notion that the CXCR2/CXCR2 ligand biological axis has a bimodal function during the course of BOS: early, it is important for neutrophil recruitment and later, during fibro-obliteration, it is important for vascular remodeling independent of neutrophil recruitment.
\end{abstract}

\section{Introduction}

The frequency of the occurrence of bronchiolitis obliterans syndrome $(\mathrm{BOS})$ is the main reason that the 5-year survival rate after lung transplantation is dismally low, at $42 \%(1,2)$. The histopathology of BOS reflects its clinical presentation with obstruction and obliteration of airways that is characterized by an infiltration of peribronchiolar leukocytes that eventually invade and disrupt the submucosa, basement membrane, and airway epithelium $(1,2)$. This is followed by fibroproliferation, granulation tissue formation, and the accumulation of ECM, ultimately ending in fibro-obliteration of the allograft airway $(1,2)$.

Angiogenesis is the formation of new blood vessels and is essential for the physiologic process of embryogenesis, tissue growth, and tumorigenesis (3-5). Angiogenesis has also been found to be central to the progression of various chronic inflammatory pathologic conditions, including diabetic retinopathy, macular degeneration, pulmonary fibrosis, and RA $(6,7)$. These diseases are characterized by chronic inflammation and fibroproliferation associated

Nonstandard abbreviations used: BALF, bronchoalveolar lavage fluid; BOS, bronchiolitis obliterans syndrome; $\mathrm{CMP}$, corneal micropocket; control $1 \mathrm{Ab}$, control $\mathrm{Ab}$ for RB6-8C5 Ab; control $2 \mathrm{Ab}$, control Ab for anti-CXCR2 Ab; CsA, cyclosporin A; CXCL8 CXC chemokine ligand 8; CXCR2, CXC chemokine receptor 2; F8Ag, factor VIII-related antigen; FBOS, future BOS; HLMVEC, human lung microvascular endothelial cell; TBOS, treated BOS.

Conflict of interest: The authors have declared that no conflict of interest exists.

Citation for this article: J. Clin. Invest. 115:1150-1162 (2005).

doi:10.1172/JCI200524233. with marked vascular remodeling. Since the fibro-obliteration process occurring during BOS is analogous to these fibroproliferative diseases, we hypothesized that vascular remodeling due to aberrant angiogenesis during fibro-obliteration of the allograft airway may contribute to the pathogenesis of BOS.

The CXC chemokine subfamily can be divided into 2 groups according to the presence $\left(\mathrm{ELR}^{+}\right)$or absence (ELR-) of an ELR (Glu-Leu-Arg) motif located immediately before the first cysteine residue at the $\mathrm{NH}_{2}$ terminus (4). $\mathrm{ELR}^{+} \mathrm{CXC}$ chemokines are known for their potent chemoattraction for neutrophils as well as their ability to promote angiogenesis (4). ELR ${ }^{+}$CXC chemokines mediate angiogenesis through the $\mathrm{G}$ protein-coupled receptor CXC chemokine receptor 2 (CXCR2) on endothelial cells (4). Furthermore, these ELR ${ }^{+}$CXC chemokines have been implicated as the dominant mediator of aberrant angiogenesis and vascular remodeling in disease processes such as adult respiratory distress syndrome (ARDS), pulmonary fibrosis, and tumor growth (8-11).

We found that lung biopsy specimens from patients with BOS had increased vascular remodeling. In addition, bronchoalveolar lavage fluid (BALF) from patients with future BOS (FBOS) (see Methods), BOS, and treated BOS (TBOS) had increased angiogenic activity. Furthermore, this angiogenic activity was predominately due to elevated levels of multiple ELR ${ }^{+}$CXC chemokines. Proofof-concept studies using a murine model of BOS also demonstrated increased vascular remodeling that paralleled $\mathrm{ELR}^{+} \mathrm{CXC}$ chemokine expression in tracheal allografts. Inhibition of these $\mathrm{ELR}^{+} \mathrm{CXC}$ chemokine interactions with CXCR2 using an anti- 


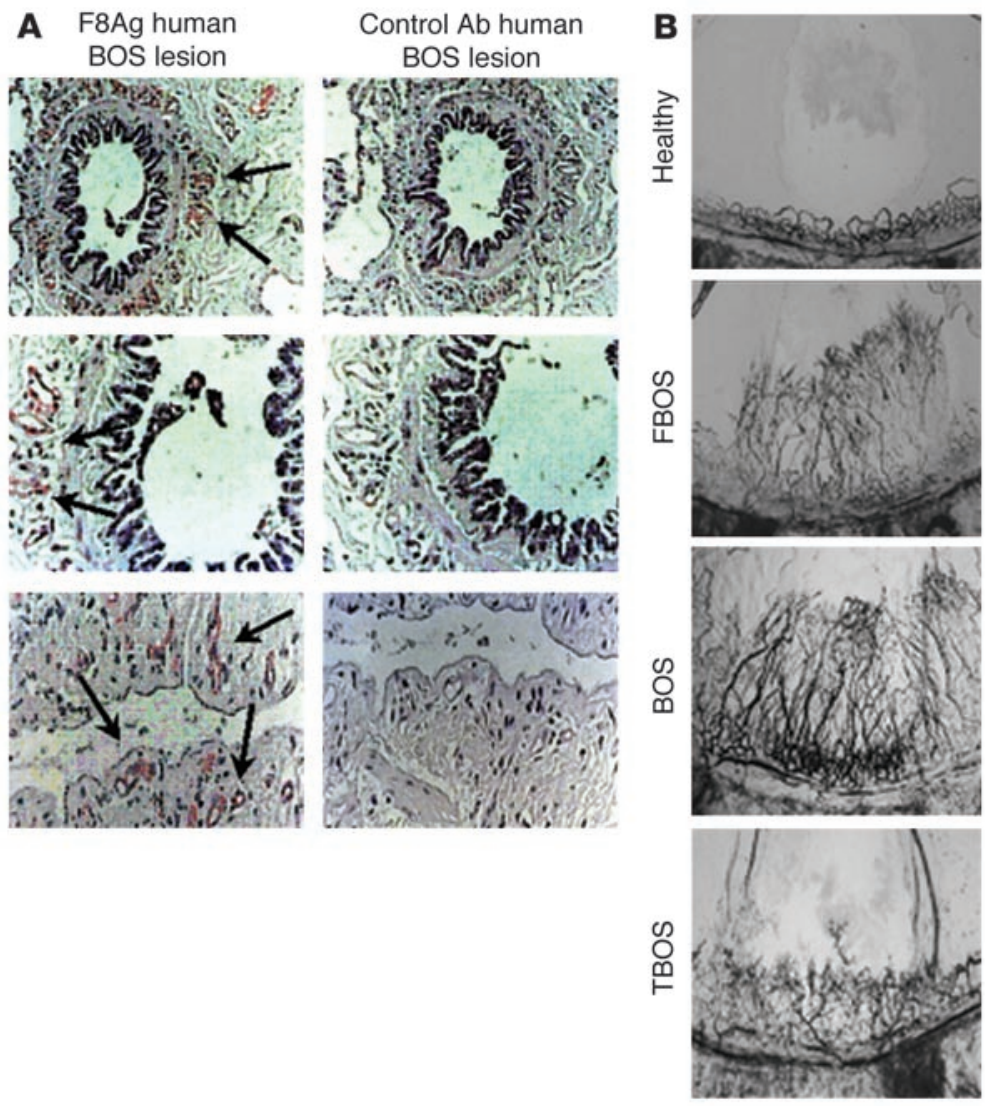

\section{Figure 1}

Vascular remodeling occurs during the pathogenesis of human BOS. (A) Representative photomicrographs of the immunolocalization of F8Ag in BOS lung tissue. Magnification, $\times 40$ (top row); $\times 100$ (middle row); and $\times 200$ (bottom row). (B) Representative photographs of corneal vascular remodeling in response to BALF specimens from healthy lung transplant recipients and recipients with FBOS, BOS, and TBOS. Magnification, $\times 20$.

in samples from healthy transplant recipients $(n=8$; that demonstrated no significant angiogenic response [0 of 6 positive CMPs]). Moreover, there was a greater angiogenic response in samples from BALF from lung transplant recipients with FBOS and TBOS $(n=8$ and $n=8 ; 5$ of 6 and 6 of 6 positive, respectively).

Elevated levels of $E L R^{+} C X C$ chemokines are associated with human BOS. Several studies have described an association among elevated levels of CXC chemokine ligand 8 (CXCL8), neutrophilia, and human BOS (12-15). However, there are multiple ELR ${ }^{+}$CXC chemokines besides CXCL8 that are not only potent neutrophil chemoattractants but are also critical in regulating angiogenesis $(4,16,17)$. We examined whether there were any significant elevations in CXCL1, CXCL3, CXCL5, CXCL7, and CXCL8 in BALF in a 4-group comparison among lung transplant recipients with BOS $(n=30)$, FBOS $(n=28)$, and TBOS $(n=10)$ and healthy lung transplant recipients $(n=47)$. The levels of CXCL7 were significantly elevated in the BOS and FBOS groups compared with the healthy transplant

CXCR2 Ab inhibited early neutrophil infiltration and late vascular remodeling and attenuated tracheal allograft fibro-obliteration. However, the effect of anti-CXCR2 Ab on the inhibition of vascular remodeling was independent of its effect on neutrophil recruitment. Moreover, CXCR2 ${ }^{-/-}$recipient mice receiving cyclosporin A (CsA) had more profound effects in reducing murine BOS as compared to CXCR2 $2^{+/}$mice receiving CsA or neutralizing CXCR2 alone. These studies demonstrate the importance of the CXCR2/ CXCR2 ligand biologic axis during the course of BOS.

\section{Results}

Aberrant angiogenesis is associated with late allograft dysfunction (BOS). We first assessed whether aberrant angiogenesis was present in human BOS by performing immunolocalization studies of factor VIII-related antigen (F8Ag) on human BOS and normal lung tissue specimens. We chose F8Ag since it is not expressed on alveolar capillary endothelium and therefore can be used to distinguish endothelium in areas of vascular remodeling from endothelium associated with collapsed alveoli. F8Ag was strongly expressed in areas surrounding BOS lesions (Figure 1A). Quantitative analysis of F8Ag-positive vessels demonstrated a marked increase in vascular remodeling of the allograft airway in individuals with BOS compared with those in whom lung biopsies were normal (Table 1). To determine whether angiogenic activity was present in the lungs of transplant recipients with BOS compared with healthy transplant recipients, we used the corneal micropocket (CMP) assay of angiogenesis. We found that there was a greater angiogenic response in pooled samples of human BALF normalized to total protein from patients with BOS ( $n=8 ; 6$ of 6 positive CMPs) than recipients (Figure $2 \mathrm{~A}$ ). In addition, the levels of CXCL3 and CXCL8 were significantly elevated in the FBOS, BOS, and TBOS groups compared with those in the healthy transplant recipients (Figure 2, B and C). No significant elevations of CXCL1 or CXCL5 were found (Figure 2, D and E). The expression of CXCL7 during FBOS and BOS and CXCL3 during FBOS, BOS, and TBOS demonstrated that 2 other ELR ${ }^{+}$CXC chemokines besides CXCL8 are involved in the pathogenesis of BOS.

BOS and FBOS BALF angiogenic activity is attributable to CXCR2/ CXCR2 ligand biology. To substantiate that the elevated levels of multiple ELR ${ }^{+}$CXC chemokines found in the BALF of patients with BOS were modulating angiogenic activity, we assessed randomly pooled samples of BALF from either recipients with BOS $(n=8)$ and healthy transplant recipients $(n=8)$ in the presence or absence of neutralizing CXCR2 or control Ab. Neutralizing Ab specific to CXCR2 was chosen, since CXCR2 has been demonstrated to be the receptor mediating $\mathrm{ELR}^{+} \mathrm{CXC}$ chemokine angiogenesis,

\section{Table 1}

Quantitative analysis of vascular remodeling in human lung allografts.

\begin{tabular}{lcc}
$\begin{array}{l}\text { Human lung } \\
\text { biopsy }\end{array}$ & $\begin{array}{c}\text { Vessel counts } \\
\text { (no. F8Ag-positive vessels/400 } \times \text { HPF) }\end{array}$ & \\
Bronchiolitis obliterans & $18.3 \pm 2.1$ & $P=0.001$ \\
Normal lung biopsy & $5.4 \pm 3.0$ & \\
\hline
\end{tabular}

HPF, high power field. 

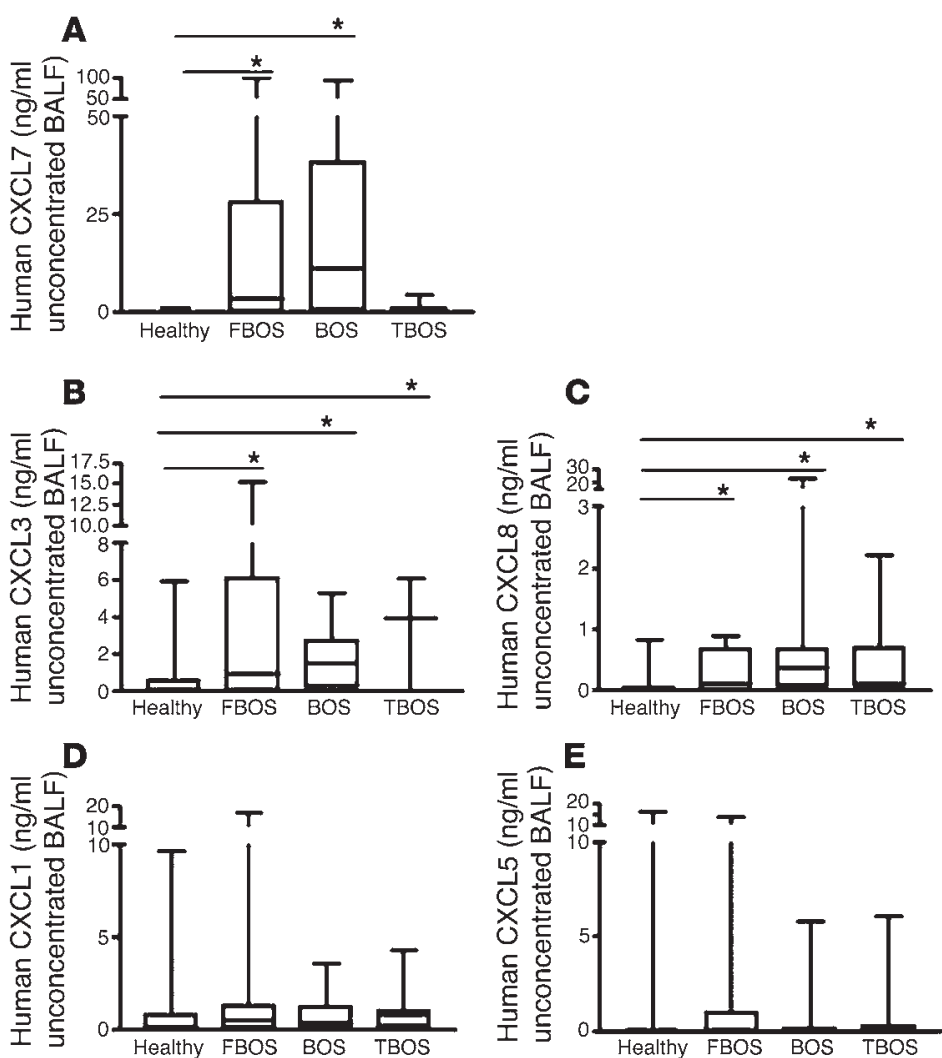

\section{Figure 2}

Human CXCL7 (A), CXCL3 (B), CXCL8 (C), CXCL1 (D), and CXCL5 (E) protein levels in unconcentrated BALF from recipients with FBOS, BOS, and TBOS and healthy lung transplant recipients in a 4-group comparison. ${ }^{*} P<0.001$.

attenuated the angiogenic activity of BALF from patients with BOS (1 of 6 positive) compared with control Ab ( 6 of 6 positive) (Figure $3 C$ ). In addition, neutralizing Ab specific to CXCR2 significantly inhibited the angiogenic activity of BALF from recipients with FBOS and TBOS (0 of 6 and 1 of 6 positive, respectively), as compared with control Abs (5 of 6 and 6 of 6 positive, respectively) (Figure 3C). To confirm our results, we performed human lung microvascular endothelial cell (HLMVEC) chemotaxis in the presence of anti-CXCR2 or control $\mathrm{Ab}$ in response to BALF from recipients with FBOS, BOS, and TBOS and healthy transplant recipients. Marked HLMVEC chemotaxis was induced in the FBOS, BOS, and TBOS groups compared with healthy lung transplant recipients (Figure 3D). Neutralizing $\mathrm{Ab}$ specific to CXCR2 significantly reduced endothelial cell chemotactic activity in the FBOS, BOS, and TBOS groups compared with control Ab (Figure 3D). There was no significant effect found with neutralizing CXCR2 or control $\mathrm{Ab}$ in the healthy group (Figure $3 \mathrm{D}$ ). These findings suggest that the $\mathrm{ELR}^{+} \mathrm{CXC}$ chemokines are angiogenic factors that mediate angiogenesis through CXCR2 during human BOS and are also associated with FBOS and TBOS patients.

$V E G F$ does not significantly contribute to the angiogenic activity in BOS. Since VEGF has been shown to be an important angiogenic factor, we assessed whether it contributes to the angiogenic activity in BOS. The levels of VEGF in BALF from patients with FBOS, BOS, and TBOS and healthy transplant recipients were not statistically different (FBOS, $0.21 \pm 0.14 \mathrm{ng} / \mathrm{ml}$; BOS, $0.14 \pm 0.10 \mathrm{ng} / \mathrm{ml}$; TBOS,
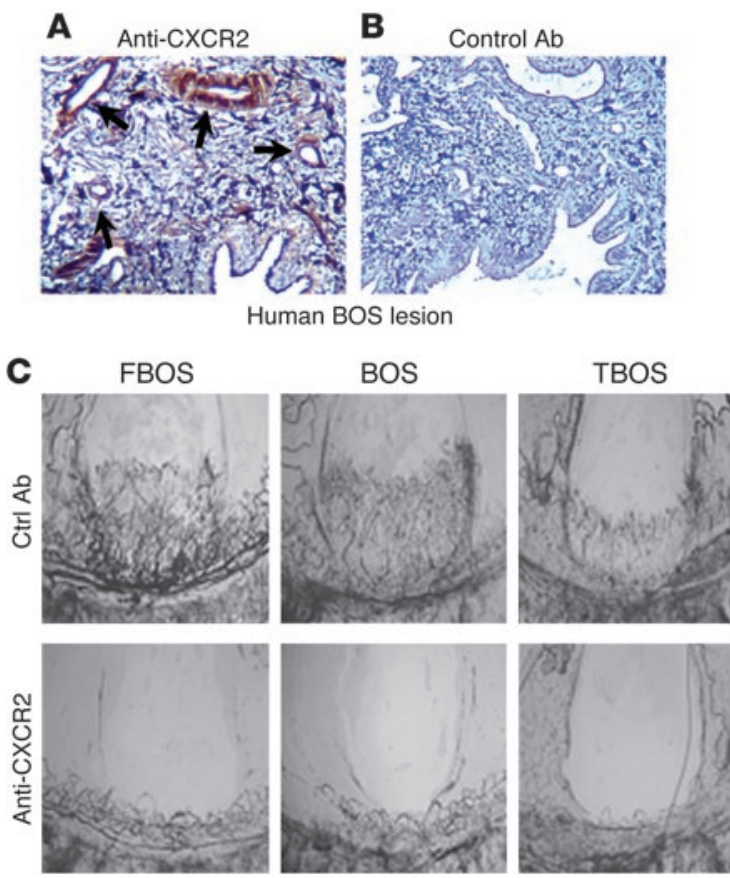

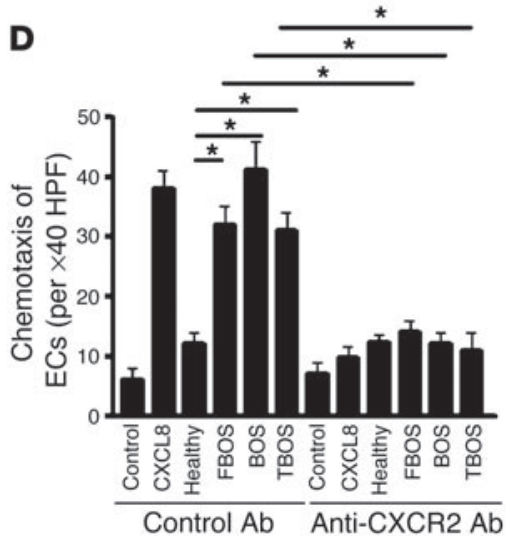

\section{Figure 3}

Representative photomicrographs of the immunolocalization of CXCR2 in human BOS lung tissue. BOS lung specimen immunostained for CXCR2 demonstrating immunolocalization to the vascular endothelium (A) and BOS lung specimen immunostained with control $\mathrm{Ab}(\mathrm{B})$. Magnification, $\times 400$. (C) Representative photomicrograph of corneal vascular remodeling in response to FBOS, BOS, and TBOS BALF specimens with neutralizing anti-CXCR2 Ab compared with control Ab. Magnification, $\times 20$. (D) BALF $\mathrm{ELR}^{+} \mathrm{CXC}$ chemokines from patients with FBOS, BOS, and TBOS are biologically angiogenic as determined by HLMVEC chemotaxis. There is significantly more chemotaxis to the FBOS, BOS, and TBOS BALF compared with that from healthy transplant recipients. Neutralizing Abs to CXCR2 inhibited chemotaxis in the FBOS, BOS and TBOS groups, but had no effect on the healthy group. ${ }^{*} P<0.05$. 
A

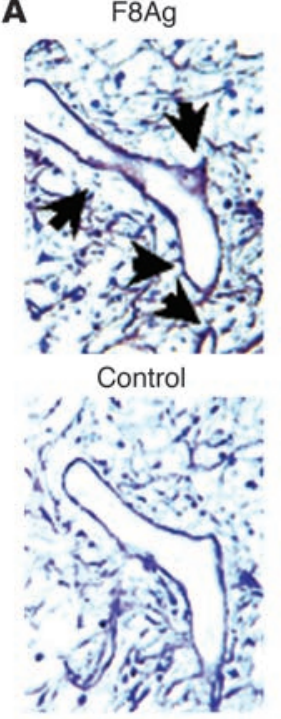

B
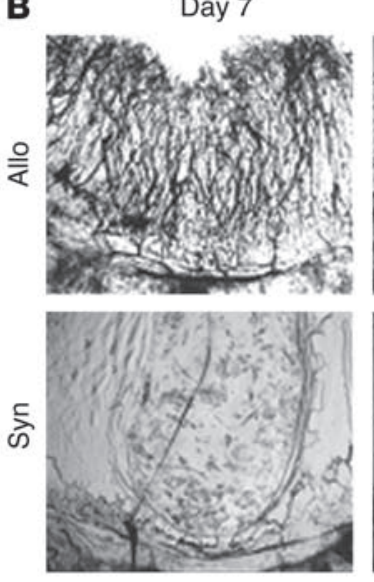

Day 21
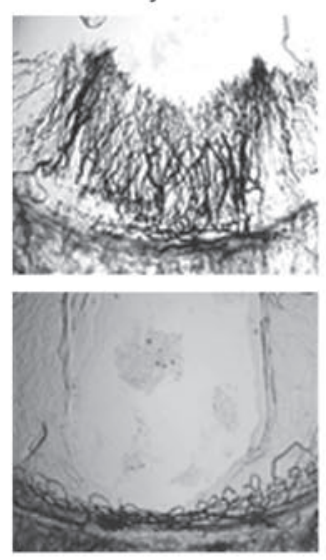

C
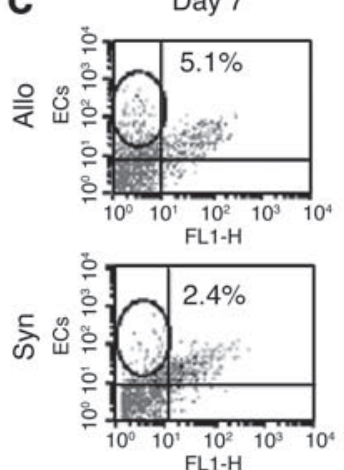

Day 21
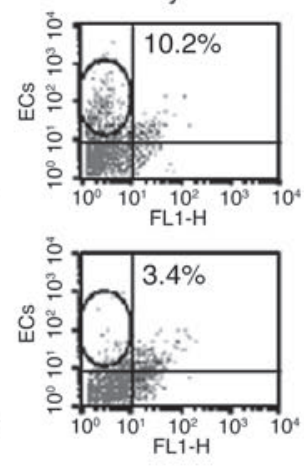

Figure 4

Vascular remodeling occurs during the pathogenesis of murine BOS. (A) Representative photomicrographs of immunolocalization of F8Ag in a murine fibro-obliteration lesion at day 21 as compared with control Ab. Magnification, $\times 400$. (B) CMP assay for vascular remodeling of tracheal-derived angiogenic activity from allografts (Allo) and syngeneic controls (Syn) at days 7 and 21. Magnification, $\times 20$. (C) FACS analysis of MECA-32 from single-cell suspension of allograft and syngeneic control digests at days 7 and 21 .

$0.04 \pm 0.02 \mathrm{ng} / \mathrm{ml}$; and healthy, $0.19 \pm 0.11 \mathrm{ng} / \mathrm{ml}$, respectively; $P>0.05)$. Furthermore VEGF angiogenic activity was evaluated using the CMP assay. Neutralization of VEGF did not significantly attenuate the angiogenic activity of BALF from recipients with BOS ( 6 of 6 positive) as compared with control Ab (5 of 6 positive). To determine whether the anti-VEGF could block VEGF-mediated angiogenesis in the CMP, we assessed VEGF-induced angiogenesis in the CMP in the presence of anti-VEGF or control Ab. Anti-VEGF attenuated VEGF-induced angiogenesis ( 0 of 6 positive) compared with control Ab ( 6 of 6 positive). These findings confirmed that BALF-derived VEGF from either BOS or FBOS patients did not play a significant role in promoting angiogenic activity.

Murine BOS tissue demonstrates angiogenic activity. Our findings from specimens from patients with BOS after lung transplantation demonstrated the following: (a) vascular remodeling is present in tissue specimens associated with BOS lesions; and (b) BALF from FBOS, BOS, and TBOS displays angiogenic activity in CMP and endothelial cell chemotaxis assays that was dependent on the CXCR2/CXCR2 ligand biological axis and not VEGF. These findings support the notion that CXCR2/CXCR2 ligands are critical factors in promoting angiogenic activity in human BOS. To ascertain whether this biology actually contributes to aberrant angiogenesis/vascular remodeling, we used an animal model of BOS as previously described $(19,20)$.

To determine whether aberrant angiogenesis was associated with murine BOS as it is with human BOS, we assessed immunolocalization of F8Ag on allograft tissue at day 21, a time point when significant fibro-obliteration occurred. F8Ag was strongly expressed on endothelial cell in areas of fibro-obliteration (Figure 4A). In addition, quantitative analysis of F8Ag-positive vessels demonstrated a marked increase in vascular remodeling in allografts as compared with syngeneic controls at days 7 and 21 (Table 2). Furthermore, angiogenic activity was assessed from tracheal allografts compared with syngeneic controls. Using the
CMP assay, we found that the angiogenic response was greater in pooled tracheal allograft homogenates at days 7 and $21(n=12$ and $n=12 ; 5$ of 6 and 6 of 6 positive, respectively) than in tracheal syngeneic control homogenates at the same time points $(n=12$ and $n=12$, respectively) ( 1 of 6 and 0 of 6 positive, respectively) (Figure 4B). To further substantiate that aberrant vascular remodeling was occurring in the allografts compared with syngeneic controls, we performed FACS analysis of the pan-endothelial cell marker MECA-32 on single-cell suspensions of tracheal digests at days 7 and 21 . We found significantly more endothelial cells in the tracheal allografts compared with syngeneic controls at both time points (Figure 4C). These data indicate that vascular remodeling is a significant component of the pathogenesis of murine BOS.

$E L R^{+}$CXC chemokine levels are elevated during murine BOS. Knowing that the CXCR2 ligands are potent inducers of angiogenesis, we assessed the kinetics of murine CXCL1 and CXCL2/3 expression during murine BOS. Real-time quantitative PCR of tracheal homogenates demonstrated markedly greater mRNA expression

\section{Table 2}

Quantitative analysis of vascular remodeling in murine tracheal allografts

$\begin{array}{lcc}\text { Murine group } & \begin{array}{c}\text { Vessel counts } \\ \text { (no. F8Ag-positive vessels/400 } \times \text { HPF) }\end{array} & P \text { value } \\ \text { Day 7 } & 9.8 \pm 2.1 & \\ \text { Allograft } & 6.6 \pm 2.3 & P=0.003 \\ \text { Syngeneic control } & & \\ \text { Day 21 } & 14.6 \pm 5.2 & P=0.001 \\ \text { Allograft } & 4.5 \pm 2.4 & \\ \text { Syngeneic control } & & \end{array}$



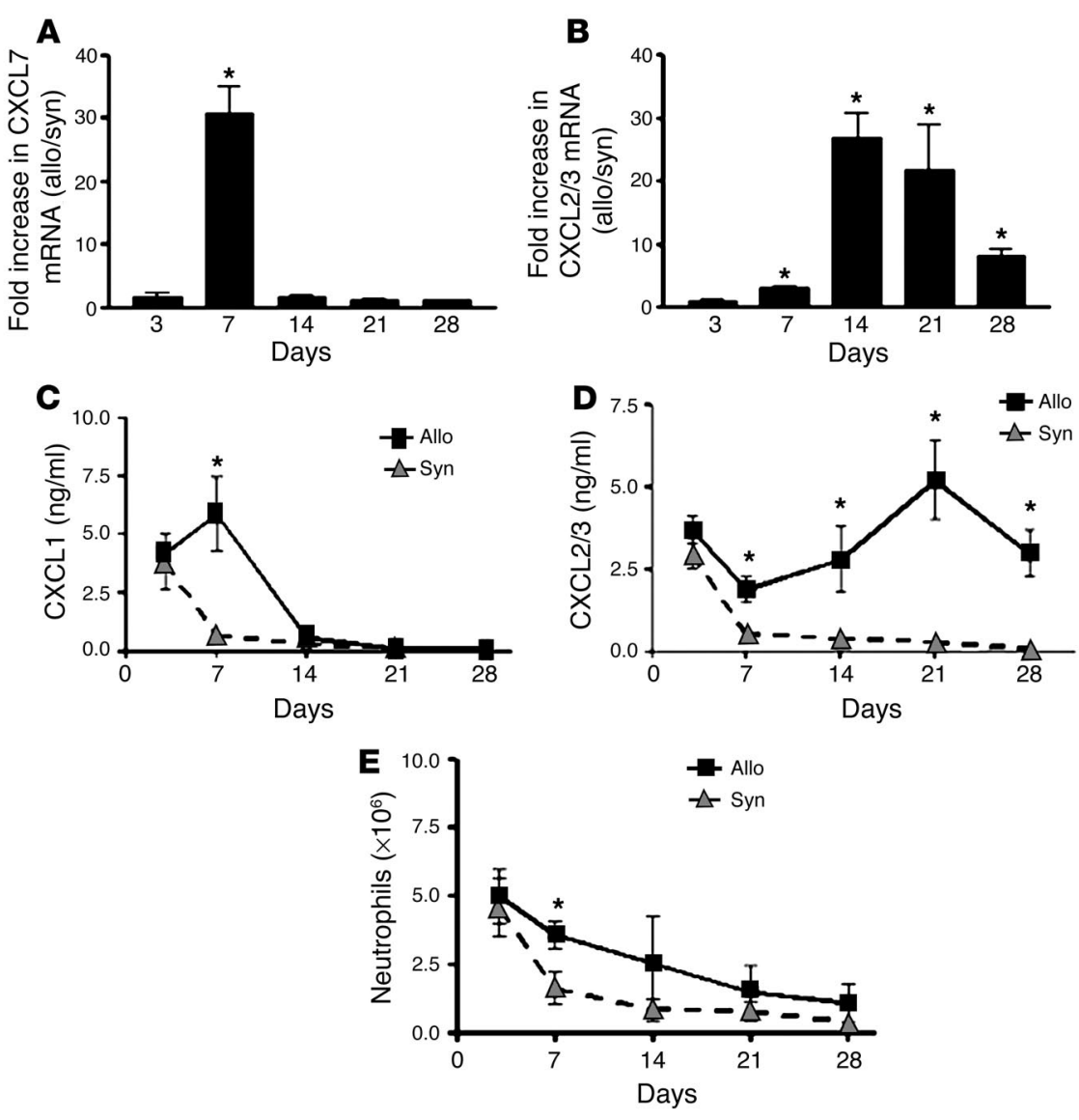

for both CXCR2 ligands from the allografts compared with the syngeneic controls (Figure 5, A and B). Interestingly, CXCL1 mRNA expression was only significantly elevated at day 7 (Figure $5 A)$. In contrast, CXCL2/3 mRNA expression was elevated at day 7 , peaked at day 14 , and remained markedly elevated throughout the remainder of the time course compared with syngeneic controls (Figure 5B). Protein levels of CXCR2 ligands were determined by specific ELISAs on tracheal homogenates over the 28 -day time course. The protein levels of CXCL1 and CXCL2/3 paralleled their mRNA expression (Figure 5, C and D). Interestingly, the pattern of neutrophil influx, as assessed by FACS analysis of single-cell suspensions from tracheal digests, demonstrated an early influx at days 3 and 7 in both allografts and syngeneic controls (Figure 5E). However after day 7, the number of infiltrating neutrophils was similar in allografts and syngeneic controls (Figure 5E). In contrast, the allografts continued to express markedly elevated levels of CXCL2/3 at days 14-28 in the absence of significant neutrophil infiltration in the allograft (Figure 5).

CXCR2 expression in the tracheal allograft parallels the production of CXCR2 ligands during murine BOS. CXCR2 is the shared receptor for all of the ELR ${ }^{+}$CXC chemokines. Real-time quantitative PCR of tracheal homogenates demonstrated a significant increase in CXCR2 mRNA expression in allografts compared with syngeneic controls (Figure 6A). CXCR2 expression peaked at day 14 and remained persistently elevated throughout the remainder of the time course, paralleling the expression CXCL2/3 to a greater extent than that of CXCL1 (Figure 5 and 6A). Interestingly, at days 14-28, when allograft neutrophil infiltration was minimal,

\section{Figure 5}

ELR+ ${ }^{+} \mathrm{CXC}$ chemokines are elevated during the pathogenesis of murine BOS. Real-time quantitative PCR of CXCL1 (A) and CXCL2/3 (B) mRNA expression presented as fold increase in chemokine expression in allografts compared with syngeneic controls at days $3,7,14,21$, and 28. Protein levels of CXCL1 (C) and CXCL2/3 (D) in allografts and syngeneic controls at days 3 through 28. (E) FACS analysis of neutrophils from tracheal allografts undergoing BOS as compared with syngeneic controls. ${ }^{*} P<0.05$.

there was a persistent and significant elevation of CXCR2 mRNA expression. Knowing that endothelial cells can express CXCR2, we performed dual-color FACS analysis on single-cell suspensions of whole tracheal digests for the cell surface expression of CXCR2 protein on endothelial cells that were positive for MECA-32. We found a significant increase in cell surface expression of CXCR2 on endothelial cells from allografts at days 7 and 21 (Figure 6B). Immunolocalization studies of CXCR2 on the allografts at day 21 confirmed these results. CXCR2 was strongly expressed on the vasculature in areas of fibroobliteration of the airway (Figure 6C). This is consistent with the hypothesis that CXCR2/ CXCR2 ligand biological function promotes a proangiogenic microenvironment during the course of BOS.

Angiogenic activity is attributable to CXCR2/CXCR2 ligand biology in murine BOS. To substantiate that the CXCR2 ligands are modulating tracheal allograft-derived angiogenic activity, we randomly pooled tracheal allografts at days 7 and $21(n=12$ and $n=12$, respectively) in the presence or absence of specific neutralizing CXCR2 or control $\mathrm{Ab}$ and assessed their angiogenic activity in the CMP. Neutralizing Ab specific to CXCR2 significantly attenuated the angiogenic activity of allografts at days 7 and 21 (0 of 6 and 1 of 6 positive, respectively) compared with control Ab (6 of 6 and 5 of 6 positive, respectively) (Figure 7A). To further analyze CXCR2/CXCR2 ligand angiogenic activity during murine BOS, we performed in vivo neutralization of CXCR2 in recipients of tracheal allografts. Mice received specific anti-CXCR2 or control Ab at days $3,5,7,9,11,13$, and 15 . We chose to initiate therapy at day 3 because it takes 3 days for the allograft to become vascularized after implantation $(19,20)$. The angiogenic activity of randomly pooled tracheal allografts harvested from animals treated with anti-CXCR $2 \mathrm{Ab}$ or control Ab at days 7 and $21(n=12$ and $n=12$, respectively) was assessed in the CMP. Tracheal allografts from animals that received in vivo neutralizing Ab specific to CXCR2 had significantly less angiogenic activity at days 7 and 21 (0 of 6 and 1 of 6 positive, respectively) compared with those from animals that received in vivo control $\mathrm{Ab}$ at days 7 and 21 (6 of 6 and 5 of 6 positive, respectively). We further confirmed this data using FACS analysis for endothelial cells on single-cell suspensions of tracheal digests at days 7 and 21. There were significantly fewer endothelial cells found in the allografts from the anti-CXCR2- compared with the control Ab-treated group (Figure 7B). These data demonstrate 


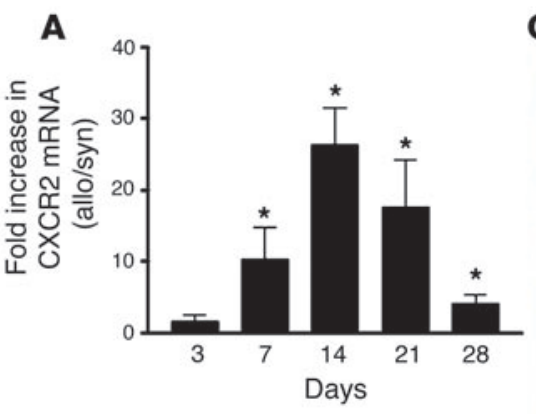

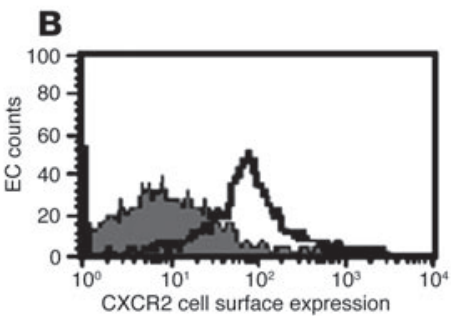

Allo (d 7)
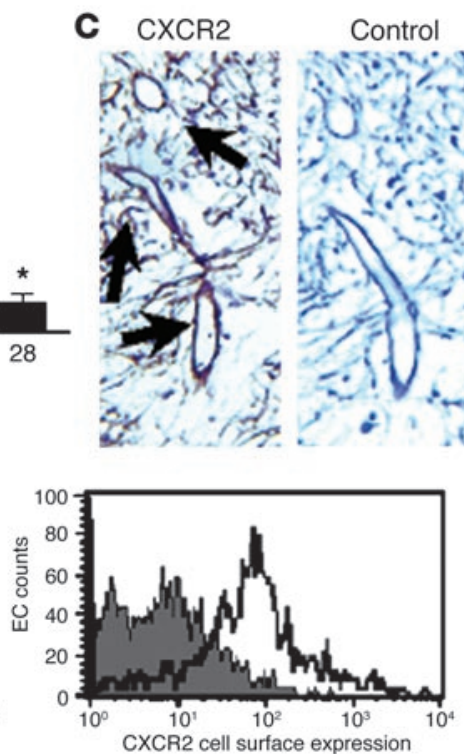

Allo (d 21) that inhibiting CXCR2/CXCR2 ligand biology has an effect on reducing vascular remodeling during the course of murine BOS.

To determine whether VEGF was contributing to the aberrant angiogenic activity seen in BOS, we measured VEGF protein levels by ELISA during the course of murine BOS. VEGF levels were significantly elevated in the allografts at day 3 (i.e., during ischemia/ reperfusion injury) compared with syngeneic controls $(2.4 \pm 0.2$ vs. $1.5 \pm 0.2 \mathrm{ng} / \mathrm{ml}$, respectively; $P<0.05)$. In addition, there was a trend toward elevated levels of VEGF in allografts at day 7 compared with syngeneic controls ( $3.3 \pm 1.1$ vs. $1.6 \pm 0.6 \mathrm{ng} / \mathrm{ml}$, respectively; $P>0.05)$. In contrast, after day $7, \mathrm{VEGF}$ levels were found to markedly decline to levels comparable to those of syngeneic controls (data not shown). Moreover, we assessed VEGF's ability to modulate tracheal allograft-derived angiogenic activity using the CMP assay. Twelve randomly pooled tracheal allografts at day 7 in the presence or absence of neutralizing anti-VEGF or control Abs were placed in the CMP. Neutralizing anti-VEGF did not significantly attenuate the angiogenic activity of allograft-treated tissue at day 7 ( 6 of 6 positive) compared with control $\mathrm{Ab}$ (5 of 6 positive). In addition, anti-VEGF blocked the activity of exogenous VEGF in this assay. These data suggest that CXCR2/CXCR2 ligands play a greater role in promoting angiogenesis than VEGF during the course of murine BOS.

Neutralization of CXCR2 inhibits angiogenic activity independently of reducing the infiltration of neutrophils during murine BOS. Besides endothelial cells, neutrophils also express CXCR2. On this basis, we next determined whether inhibiting CXCR2/CXCR2 ligand biology decreases neutrophil recruitment during murine BOS. In vivo

\section{Figure 7}

Vascular remodeling and angiogenic activity during murine BOS is attributable to CXCR2/CXCR2 ligand biology. (A) Representative CMP assay for vascular remodeling of tracheal-derived angiogenic activity from allografts with ex vivo-administered anti-CXCR2 Ab compared with control Ab. Magnification, $\times 20$. (B) FACS analysis of MECA-32 from allografts treated with in vivo anti-CXCR2 Ab compared with control $\mathrm{Ab}$.

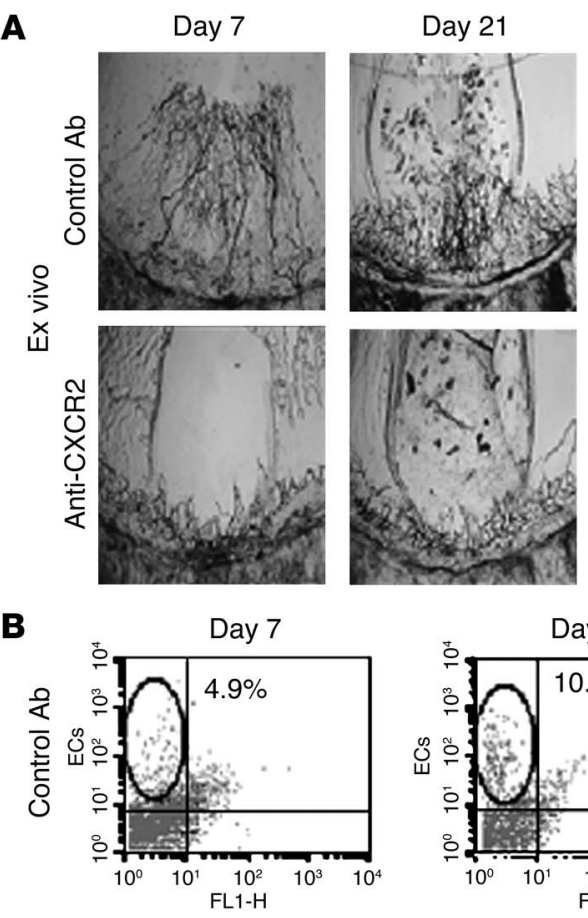

neutralization of endogenous murine CXCR2, as compared with control $\mathrm{Ab}$, was performed as described above. Kinetics of FACS analysis on single-cell suspensions of whole tracheal digests demonstrated that neutralizing anti-CXCR2 Ab reduced neutrophil infiltration at day 7 (Figure 8A). However, by days 14 and 21 neutralizing anti-CXCR2 had no significant effect on neutrophil infiltration. Based on these findings, the reduction in angiogenic activity using antiCXCR2 Ab during murine BOS could be a result of inhibition of the early influx of neutrophils into the allograft (i.e., by reducing the numbers of infiltrating neutrophils and their potential expression of proangiogenic mediators). To explore this possibility, we performed neutrophil depletion studies in vivo during murine BOS. Neutrophil depletion was achieved by i.p. administration of RB6-8C5 Ab to recipients of tracheal

A
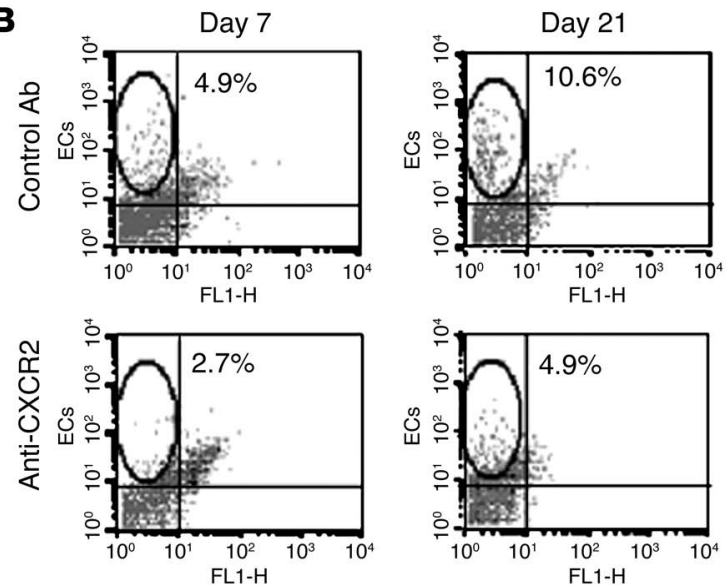

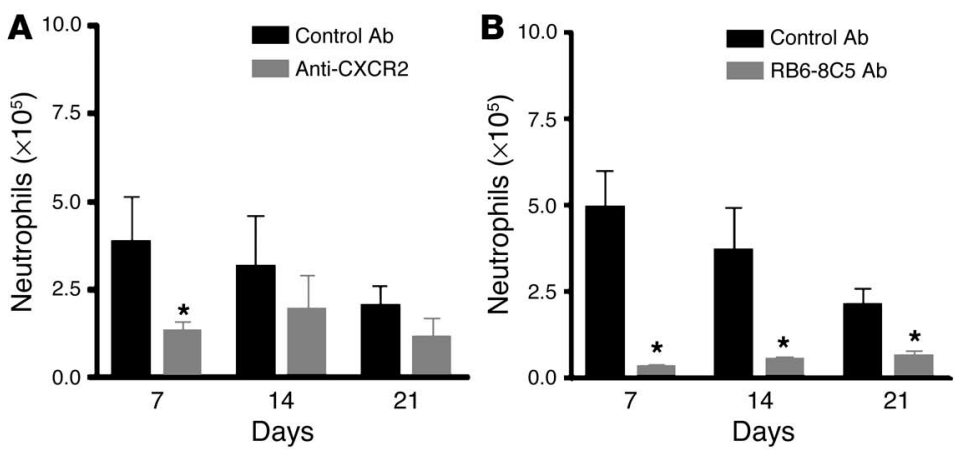

C
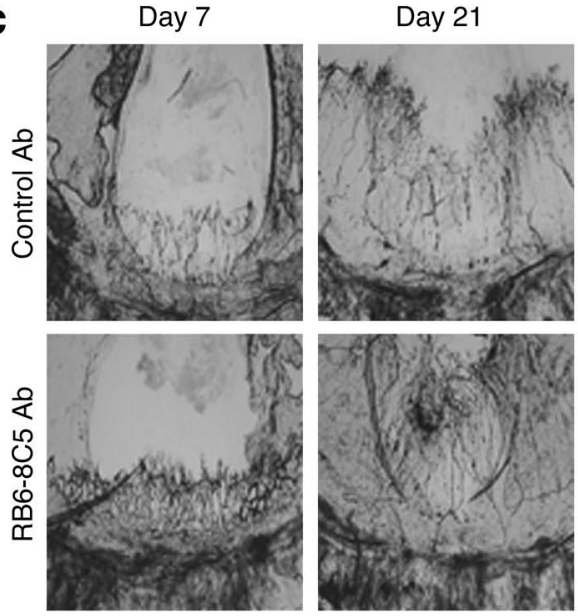

D
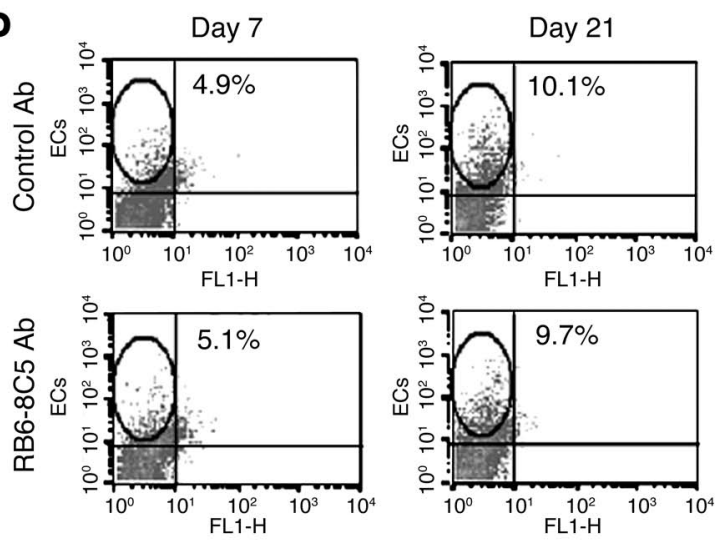

allografts starting at 1 day before transplantation and given every other day until day 17, compared with control $\mathrm{Ab}$ given every other day until day 17. The use of RB6-8C5 resulted in peripheral blood neutropenia. FACS analysis of single-cell suspensions of whole tracheal digests demonstrated a significant reduction of infiltrating neutrophils in animals treated with RB6-8C5 Ab compared with control $\mathrm{Ab}$ at all time points evaluated (Figure $8 \mathrm{~B}$ ). We used the CMP assay to determine the effects of neutrophil depletion on angiogenic activity in tracheal allografts from recipients treated in vivo with RB6-8C5 Ab compared tracheal allografts from recipients treated with control $\mathrm{Ab}$ at days 7 and 21 . We found that a similar angiogenic response was induced in pooled tracheal allograft homogenates from animals treated with RB6-8C5 Abs at days 7 and 21 ( $n=12$ and $n=12 ; 5$ of 6 and 6 of 6 positive, respectively) compared with tracheal allografts treated with control Abs $(n=12$

\section{Figure 8}

Inhibition of CXCR2/CXCR2 ligand biology attenuates vascular remodeling and angiogenic activity independent of its effects on neutrophils during murine BOS. (A) FACS analysis of neutrophils in tracheal allografts from recipients treated with anti-CXCR2 compared with control Ab. (B) FACS analysis of neutrophils in tracheal allografts from recipients treated with RB6-8C5 Ab compared with control Ab. ${ }^{*} P<0.05$. (C) Representative CMP assay for vascular remodeling of tracheal-derived angiogenic activity from allografts with in vivo-administered RB6-8C5 Ab compared with control $\mathrm{Ab}$ at days 7 and 21. Magnification, $\times 20$. (D) FACS analysis of MECA-32 from allografts treated in vivo with RB6-8C5 Ab compared with control Ab.

and $n=12 ; 6$ of 6 and 6 of 6 positive, respectively) (Figure $8 \mathrm{C})$. To further analyze the effects of neutrophil depletion on vascular remodeling, we also performed FACS analysis of endothelial cells using MECA-32 on single-cell suspensions of whole tracheal allograft digests at days 7 and 21 . There was no significant difference in allograft endothelial cells at either time point (Figure 8D). These results demonstrate that vascular remodeling of the tracheal allograft during the course of murine BOS occurs independently of infiltrating neutrophils within the allograft.

Inbibition of CXCR2/CXCR2 ligand biology results in the attenuation of murine BOS. With a significant reduction in angiogenic activity of the allografts from animals treated with anti-CXCR2 Ab, we next assessed whether there was any effect on the pathology of murine BOS. We used a quantitative overall cumulative BOS score (see Methods) based on 4 easily identifiable pathologic processes: airway lining epithelial loss, leukocyte infiltration, ECM deposition, and luminal obliteration, as previously described (19, 20). Histopathologic analysis of tracheal allografts from recipient animals treated with anti-CXCR2 demonstrated marked reductions in overall cumulative BOS scores and hydroxyproline levels compared with animals treated with control Ab throughout day 21 (Figure 9, A-C). To exclude the possibility that the effect of anti-CXCR2 was related to neutrophils, we implanted animals with tracheal allografts and exposed them to either RB6-8C5 Ab or control Ab. While there was a significant reduction in overall cumulative score at day 7, there was no effect of neutrophil depletion on cumulative BOS scores and hydroxyproline levels at days 14 and 21 (Figure 10, A-C).

To further establish the role of CXCR2/CXCR2 ligand biological axis in neutrophil recruitment in promoting angiogenesis during BOS, we next compared the effects of: anti-CXCR2 Ab plus RB6-8C5 Ab; anti-CXCR2 Ab plus control $1 \mathrm{Ab}$ (control Ab for RB6-8C5 Ab); control $2 \mathrm{Ab}$ (control Ab for anti-CXCR2 Ab) plus RB6-8C5 Ab; and control $1 \mathrm{Ab}$ plus control $2 \mathrm{Ab}$. We found a similar significant reduction in overall cumulative BOS scores and hydroxyproline levels in the anti-CXCR2 Ab plus anti-RB6-8C5 Ab group and the anti-CXCR2 Ab plus control $1 \mathrm{Ab}$ group compared with the other groups throughout the 21-day time course (Figure 11, A and B). However, the RB6-8C5 Ab plus control $2 \mathrm{Ab}$ group only demonstrated a significant reduction in overall cumulative score at day 7 (i.e., the early allograft dysfunction phase) and not days 14 and 21 (i.e., late allograft dysfunction, BOS) (Figure 11A). These findings demonstrate a critical role for CXCR2/CXCR2 
A

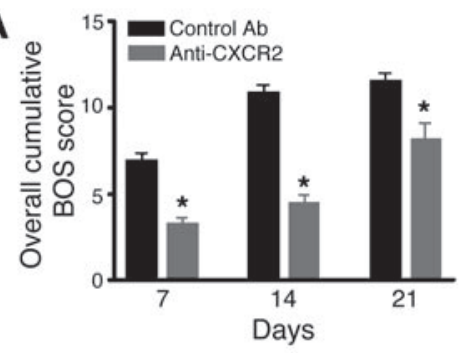

B

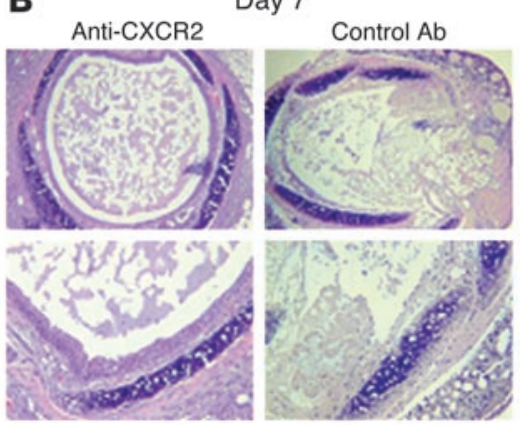

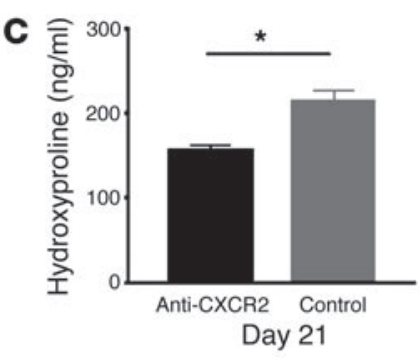

Day 21

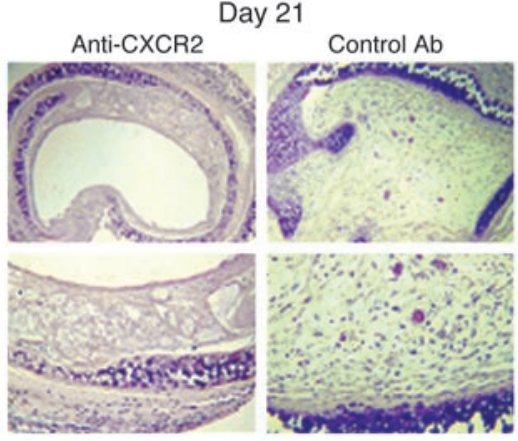

Figure 9

Inhibition of CXCR2/CXCR2 ligand biology attenuates murine BOS. (A) Quantitative analysis of histopathologic sections of tracheal allografts from animals treated with anti-CXCR2 Ab compared with control Ab. (B) Representative photomicrographs of the histopathology of tracheal allografts from animals treated with anti-CXCR2 Ab compared with control Ab at days 7 and 21 . Magnification, $\times 40$ (top row) and $\times 100$ (bottom row). (C) Hydroxyproline levels in tracheal allografts from animals treated with anti-CXCR2 $\mathrm{Ab}$ compared with control $\mathrm{Ab}$ at day $21 .{ }^{*} P<0.05$.

CsA $(20 \mathrm{mg} / \mathrm{kg} / \mathrm{d})$ and depletion of CXCR2. In these experiments CXCR2 $2^{-/}$or CXCR2 $2^{+/+}$mice on a C57BL/6 background were recipients of tracheas from $\mathrm{BALB} / \mathrm{c} \mathrm{CXCR} 2^{+/+}$mice. The recipient mice were exposed to CsA every day beginning at day 0 until the animals were sacrificed at specified time points. We found marked reductions in overall cumulative BOS scores and hydroxyproline levels in allografts from CXCR $2^{-/}$ recipients treated with CsA through day 28 com-

ligand biological axis in mediating vascular remodeling during the course of the fibro-obliterative phase of murine BOS independent of its effects on neutrophils.

The combination of inhibition of CXCR2/CXCR2 ligand biology using CXCR2 $-/-$ recipients and CsA is synergistic for attenuating murine BOS: $\mathrm{T}$ cells and other mononuclear cells are involved in the pathogenesis of BOS. Calcineurin inhibitors such as CsA predominately affect $\mathrm{T}$ cell function and have contributed to increase survival of lung allografts (21). However, with the significant side effects related to these reagents, any reduction in their dose would be important for the diminution of transplant recipient morbidity (21). For these reasons, we titrated $(5,10,15$, 20 , and $25 \mathrm{mg} / \mathrm{kg} / \mathrm{d}$ ) the lowest dose of CsA required to inhibit BOS at day 7 , the time when significant leukocyte infiltration occurs. The dose of $20 \mathrm{mg} / \mathrm{kg} / \mathrm{d}$ reduced overall cumulative BOS scores compared with the control oil-treated group at day 7. However, by day 14 , no significant reduction in overall cumulative BOS scores was detected in the CsA-treated group compared with the control (oil) vehicletreated group. With this low dose of CsA attenuating BOS only at day 7 , separate experiments were designed to evaluate the combination of

\section{Figure 10}

Neutrophil depletion does not affect late fibro-obliteration during the pathogenesis of murine BOS. (A) Quantitative analysis of histopathologic sections of tracheal allografts from animals treated with RB68C5 Ab compared with control Ab. (B) Representative photomicrographs of the histopathology of tracheal allografts from animals treated with RB6$8 \mathrm{C} 5 \mathrm{Ab}$ compared with control Ab at days 7 and 21 . Magnification, $\times 40$ (top row) and $\times 100$ (bottom row). (C) Hydroxyproline levels in tracheal allografts from animals treated with RB6-8C5 Ab compared with control $\mathrm{Ab}$ at day $21 .{ }^{*} P<0.05$.
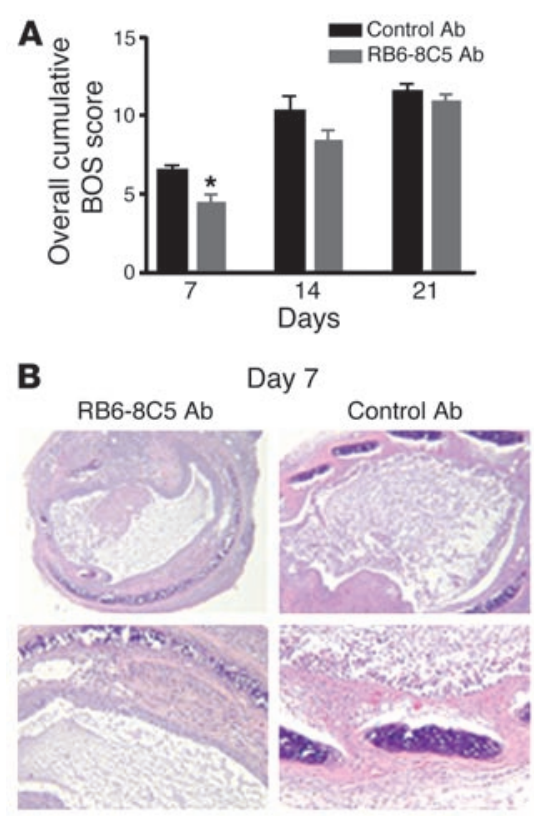
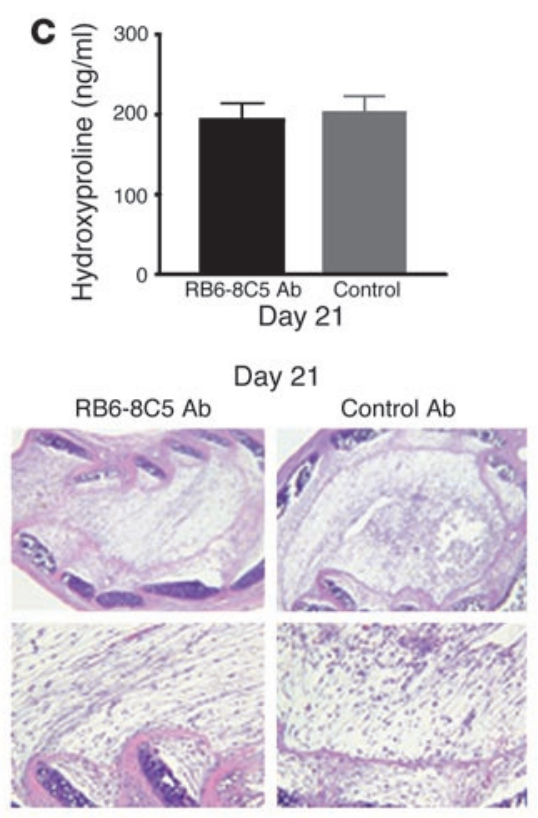


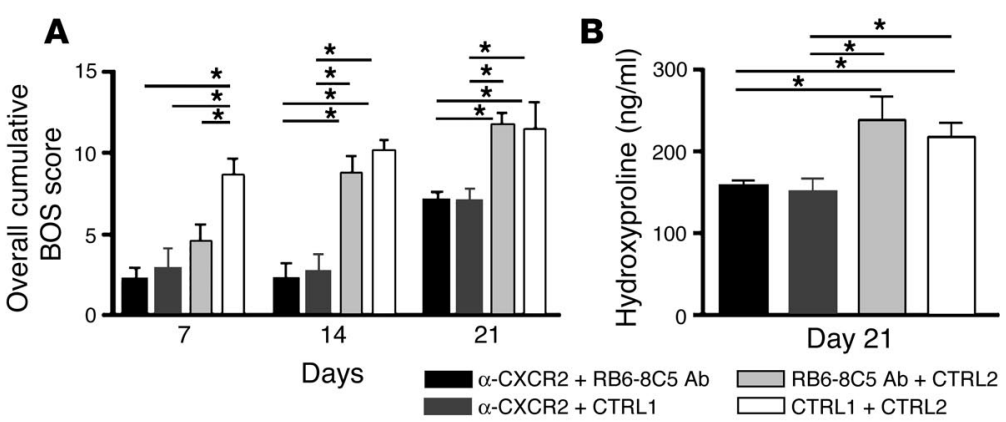

\section{Figure 11}

Neutrophil depletion plus anti-CXCR2 Ab treatment has no synergistic or additive effect on murine BOS as compared to anti-CXCR2 treatment alone. (A) Quantitative analysis of histopathologic sections of tracheal allografts from animals treated with: anti-CXCR2 Ab plus control $1 \mathrm{Ab}$ (CTRL1); anti-CXCR2 Ab plus RB6-8C5 Ab; RB6-8C5 Ab plus control $2 \mathrm{Ab}$ (CTRL2); and control $1 \mathrm{Ab}$ plus control $2 \mathrm{Ab}$. (B) Hydroxyproline levels in tracheal allografts from animals treated with: anti-CXCR2 Ab plus control $1 \mathrm{Ab}$; anti-CXCR2 Ab plus RB6-8C5 Ab; RB6-8C5 Ab plus control $2 \mathrm{Ab}$; and control $1 \mathrm{Ab}$ plus control $2 \mathrm{Ab}$. ${ }^{*} P<0.0083$. cells; this results in aberrant vascular remodeling in the context of the formation of granulation tissue around and within the allograft airway, the hallmark of BOS.

In this study, significant aberrant angiogenesis was demonstrated in lung biopsy specimens from patients with BOS. In addition, BALF from patients with FBOS, BOS, and TBOS had significant angiogenic activity. This is consistent with the findings that vascular remodeling is associated with the fibroplasia of ARDS and the pannus formation involved in RA $(7,10)$. However, to our knowledge, this is the first study to describe an association of vascular remodeling and angiogenic activity in human BOS.

Since $\mathrm{ELR}^{+} \mathrm{CXC}$ chemokines are potent regulators of angiogenesis, we evaluated multiple $\mathrm{ELR}^{+} \mathrm{CXC}$ chemokines in BALF from patients with BOS. There were marked elevations in CXCL7 levels in the FBOS and BOS groups and CXCL3 and CXCL8 levels in the FBOS, BOS, and TBOS groups. This demonstrates an association among multiple $\mathrm{ELR}^{+} \mathrm{CXC}$ chemokines and angiogenic activity in BALF from patients with FBOS, BOS, and TBOS and expands upon previous data demonstrating an association between $1 \mathrm{ELR}^{+}$CXC chemokine, CXCL8, and BOS (13, 15, 22-24). Furthermore, the elevated levels of multiple $\mathrm{ELR}^{+} \mathrm{CXC}$ chemokines in the FBOS group suggest the possibility that a persistent, very low-grade allospecific injury to the allograft airways may be promoting the production of these chemokines and eventually leads to vascular remodeling, which is required to support the fibroplasia of BOS. This concept is supported by recent studies demonstrating that minimal acute lung allograft rejection, if left untreated, leads to high-grade acute rejection/ lymphocytic bronchiolitis and eventual BOS (25). Alternatively, we cannot completely rule out the possibility of occult infections as a possible etiology. Moreover, our current treatment of BOS was not able to suppress the $\mathrm{ELR}^{+} \mathrm{CXC}$ chemokine expression and had no effect on

\section{Figure 12}

$\mathrm{CXCR2}^{-1-}$ recipients of tracheal allografts treated with CsA attenuates murine BOS for a prolonged period of time. (A) Quantitative analysis of histopathologic sections of tracheal allografts from $\mathrm{CXCR}^{-1-}$ animals treated with CsA compared with $\mathrm{CXCR}^{+/+}$animals treated with CsA. (B) Representative photomicrographs of the histopathology of tracheal allografts from $\mathrm{CXCR} 2^{--}$animals treated with CsA compared with $\mathrm{CXCR} 2^{+/+}$animals treated with CsA at day 28, Magnification, $\times 40$ (top row) and $\times 100$ (bottom row). (C) Hydroxyproline levels in tracheal allografts from CXCR2 $2^{--}$ animals treated with CsA compared with $\mathrm{CXCR} 2^{+/+}$animals treated with CsA at day 28. ${ }^{*} P<0.05$.
BOS, which suggests that one problem with this therapy may be its inability to suppress these chemokines, which are responsible, in part, for promoting angiogenesis and perpetuating BOS.

The fact that all ELR ${ }^{+} \mathrm{CXC}$ chemokines mediate angiogenesis highlights the importance of identifying a common receptor. This would provide the unique opportunity to target a putative receptor for $\mathrm{ELR}^{+} \mathrm{CXC}$ chemokine-induced angiogenesis in the context of BOS. Recent studies have demonstrated CXCR2, not CXCR1, to be the receptor that mediates angiogenic activity (18, 26-32). Our data also demonstrate that the vascular remodeling associated with BOS lesions involves endothelial cells expressing CXCR2. This is analogous to the expression of CXCR2 on endothelial cells seen in tissue surrounding human cancers, pulmonary fibrosis, and wound granulation tissue $(10,11,18,27,28,33,34)$. Based on these previous studies, we evaluated the role of elevated ELR ${ }^{+} \mathrm{CXC}$ chemokines in promoting angiogenic activity during FBOS, BOS, and TBOS. We found that the angiogenic activity in BALF from patients with FBOS, BOS, and TBOS was predominately mediated by the CXCR2/CXCR2 ligand biological axis. These data are supported by studies demonstrating that the vascular remodeling of RA and pulmonary fibrosis is principally mediated by the CXCR2/ CXCR2 ligand biological axis $(7,8,35)$. Interestingly, VEGF was not elevated in BALF from patients with FBOS, BOS, and TBOS, and anti-VEGF Ab did not inhibit angiogenic activity in BALF from patients with BOS. This confirms the results of previous studies

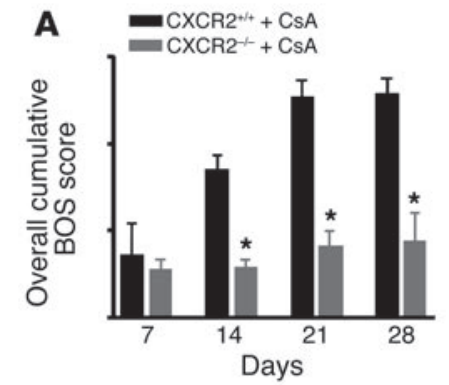

C

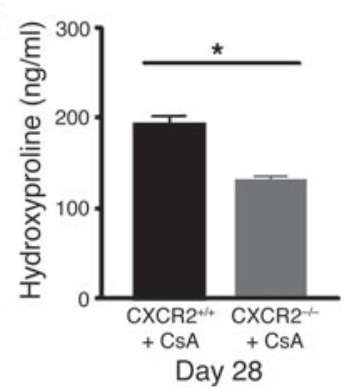

B

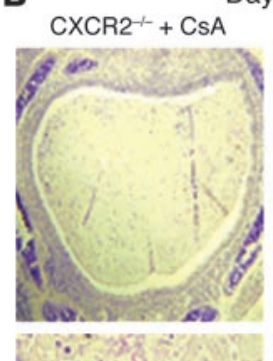

Day 28

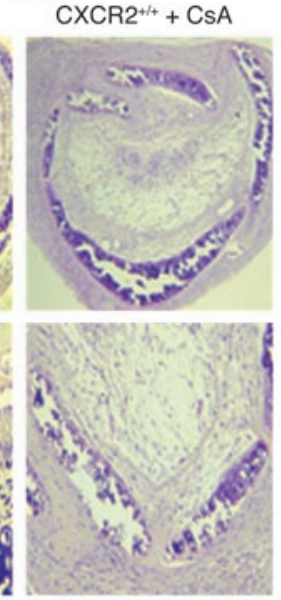


on humans, in which VEGF was not found to be associated with BOS (36), and the findings suggest a more important role for the $\mathrm{ELR}^{+} \mathrm{CXC}$ chemokines compared with VEGF in propagating vascular remodeling during the continuum of FBOS to BOS. Collectively, these studies demonstrate an important association among augmented levels of $\mathrm{ELR}^{+} \mathrm{CXC}$ chemokines, angiogenic activity, and vascular remodeling during the course of human fibroproliferative/obliterative diseases.

Given the importance of CXCR2/CXCR2 ligands in promoting angiogenic activity in humans, we next performed proof-of-concept studies using the heterotopic murine model of BOS. While this model has limitations due to its heterotopic positioning, which thus discounts the influence of adjacent airway mucosa, it has histopathologic features similar to those of human BOS $(19,20,37)$. Importantly, we found significant vascular remodeling and angiogenic activity in tracheal allografts compared with syngeneic controls, which led us to evaluate murine ELR ${ }^{+}$CXC chemokines. Both CXCL1 and CXCL2/3 levels were elevated in the tracheal allografts compared with the syngeneic controls. Similarly, the level of CXCR2, the only murine $\mathrm{ELR}^{+} \mathrm{CXC}$ chemokine receptor, was also markedly elevated in allografts. However, there was only a marked intragraft neutrophil infiltration at days 3 and 7 , the time points consistent with ischemia/ reperfusion injury phase in this model. Therefore, it was anticipated that the CXCR2 and CXCR2 ligand levels would be elevated at days 3 and 7. However it was surprising to find that CXCR2 and CXCR2 ligand levels remained persistently elevated throughout the remainder of the time course (i.e., the fibroproliferative phase), even when minimal intragraft neutrophil infiltration was present. The kinetics of CXCR2 and CXCR2 ligands suggest that ELR ${ }^{+}$CXC chemokines have an alternative function besides neutrophil recruitment during the fibroproliferative phase of BOS.

One alternative function of the $\mathrm{ELR}^{+} \mathrm{CXC}$ chemokines is the promotion of angiogenesis (4). Thus the angiogenic potential of these chemokines was assessed during murine BOS. Similar to our human data, we found that inhibition of the CXCR2/CXCR2 ligand biological axis with anti-CXCR2 both ex vivo or in vivo markedly reduced the angiogenic activity in the tracheal allografts. This was further confirmed by in vivo neutralization of CXCR2, which resulted in a reduction in endothelial cells in tracheal allografts from animals treated with anti-CXCR2 Ab. These data are supported by other studies involving models of lung cancer or pulmonary fibrosis in which inhibition of $\mathrm{ELR}^{+} \mathrm{CXC}$ chemokines lead to a reduction in angiogenesis $(8,11,35)$.

VEGF was also evaluated during murine BOS. While elevated levels of VEGF were found at the earliest time point evaluated (i.e., day 3 ), at later time points, levels were similar in both allografts and syngeneic controls, and VEGF was found to contribute little if at all to the angiogenic activity of tracheal allografts during the course of BOS. These data are in agreement with several studies demonstrating that in certain disease processes, the CXCR2/CXCR2 ligand biological axis is the major inducer of angiogenesis. For instance, in studies using a murine single lung ligation model in the absence of hypoxemia, there was a marked angiogenic response associated with elevated levels of the ELR ${ }^{+} \mathrm{CXC}$ chemokines and not $\operatorname{VEGF}(9,38)$. Similarly in a SCID xenograft model of human glioblastoma, downregulation of the tumor suppressor gene ING4 led to the upregulation of CXCL8-mediated, but not VEGF-mediated, angiogenesis (9). These studies demonstrate that in specific tumors as well as fibroproliferative diseases, $\mathrm{ELR}^{+} \mathrm{CXC}$ chemokines can be the predominate mediators of angiogenesis.
Based on the finding of a reduction in angiogenesis in tracheal allografts from animals treated with the anti-CXCR2 Ab, murine BOS was evaluated. Tracheal allografts from the animals treated with anti-CXCR2 had significant reductions in BOS scores and hydroxyproline levels. This is similar to the findings in murine models of pulmonary fibrosis, where inhibition of vascular remodeling led to a reduction in fibrosis (8). However, the anti-CXCR2 $\mathrm{Ab}$ did cause a reduction in neutrophils during the early (ischemia/ reperfusion injury) phase of allograft dysfunction. In addition, other studies have demonstrated that depletion of neutrophils or using mice deficient in the src family kinases (Hck and Fgr), which have alterations in granulocyte migration, results in inhibition of angiogenesis by reducing the expression of VEGF (39, 40). These studies suggest that the paracrine production of VEGF from neutrophils is responsible for angiogenesis. On this basis, we performed neutrophil depletion experiments to specifically determine the effect on angiogenesis during murine BOS. There was a reduction in allograft damage only at day 7 , consistent with previous data demonstrating that neutrophils are important during early allograft (ischemia/reperfusion injury) dysfunction (41-43). In contrast, neutrophil depletion did not affect vascular remodeling or fibro-obliteration. This demonstrates that the angiogenic activity of the allografts was independent of infiltrating allograft neutrophils but was dependent on the CXCR2/CXCR2 ligand biological axis. This was confirmed by the finding that there was no synergistic or additive effect of neutrophil depletion plus anti-CXCR2 Ab treatment compared with inhibition of CXCR2 alone. Collectively these studies suggest that the effects of the CXCR2/CXCR2 ligand biological axis are bimodal during BOS: early, it effects neutrophil recruitment (i.e., during the ischemia/ reperfusion injury phase), and later, it effects vascular remodeling and angiogenesis (i.e., during the fibroproliferative phase) independently of neutrophil recruitment.

Last, we determined the effects of combining CsA treatment with the inhibition of CXCR2 interactions with its ligands. We chose CXCR2 ${ }^{-/}$recipients to avoid serum sickness in wild-type mice due to prolonged use (i.e., 28 days) of the anti-CXCR2 Ab in combination with CsA and found a reduction in collagen deposition and BOS. In fact, these are the best results we have seen as of yet in this model. These findings demonstrate that the combination of inhibiting angiogenesis and immunosuppression may be a novel way to protect lung allografts from BOS.

In conclusion, we have demonstrated that multiple $\mathrm{ELR}^{+} \mathrm{CXC}$ chemokines are associated with human BOS. In addition, these chemokine interactions with their shared receptor, CXCR2, are important inducers of angiogenic activity during human BOS. Proof-of-concept studies using the murine model of BOS demonstrated that these $\mathrm{ELR}^{+} \mathrm{CXC}$ chemokines have a bimodal function. Acutely, they are responsible for intragraft neutrophil infiltration during ischemia/reperfusion injury, and chronically, they are responsible for the vascular remodeling needed to support the fibroproliferative phase of the disease independent of neutrophil infiltration. The findings of this study may ultimately result in novel therapies designed to attenuate the CXCR2/CXCR2 ligand biological axis and lead to better intervention in the prevention and treatment of BOS.

\section{Methods}

Patient population. With Institutional Review Board (IRB) approval (David Geffen School of Medicine at UCLA) and informed written consent, we 


\section{Table 3}

Time in months for each group after lung transplantation

$\begin{array}{lcc}\text { Group } & \text { Duration after lung transplantation }(\mathbf{m o}) & \boldsymbol{P} \text { value } \\ \text { H } & 19.3 \pm 2.9 & \\ \text { FBOS } & 20.2 \pm 3.6 & \text { NS } \\ \text { BOS } & 27.7 \pm 4.4 & \\ \text { TBOS } & 28.1 \pm 9.3 & \end{array}$

Nonsignificant differences in a 4-group comparison of healthy lung transplant recipients $(\mathrm{H})$ and lung transplant recipients with FBOS, $\mathrm{BOS}$, and TBOS.

prospectively enrolled all patients undergoing lung transplantation from June 1992 to April 2000. Patients were eligible for this study if they survived at least 6 months after transplantation. One hundred and sixty-nine patients were evaluated, of which 47 , who had never had an episode of acute rejection or BOS, met criteria for being healthy lung transplantation recipients; and 30 met criteria for having BOS and for whom clinical or laboratory data demonstrated no allograft colonization or infection. All transplantation recipients were routinely followed according to a standard protocol. This protocol included clinical visits weekly for the first 3 weeks, then at 6 weeks, and then at 3, 6, 9, and 12 months. Subsequently, they were seen every 4 months for the second year and thereafter annually. The clinic visit involved a history, physical examination, pulmonary function testing, and a bronchoscopy. Bronchoscopy was also performed at times when either infection or rejection was suspected. We chose the most recent available BALF samples in the healthy group to try to ensure an effective comparison of duration after transplantation between the healthy transplant recipients and patients with BOS. As shown in Table 3, there was no significant difference in the duration in months after lung transplantation between the 4 groups.

The third group evaluated was a subpopulation of the BOS group called FBOS $(n=28)$. For this group of BOS patients, BALF samples were taken from the last bronchoscopy without colonization, infection, acute rejection, or BOS. The fourth group, TBOS $(n=10)$, were BOS recipients that had undergone treatment with either pulse methylprednisolone, monoclonal OKT3, or anti-thymocyte globulin Ab; and had undergone clinical evaluation with pulmonary function testing (PFT) and bronchoscopy within 6 weeks of the treatment. Unfortunately, none of the patients treated for BOS had a response, as demonstrated by clinical parameters and by PFT criteria.

BALF was obtained from healthy lung transplant recipients and those with FBOS, BOS, TBOS by methods previously described $(19,44,45)$. The cell-free solution was aliquoted and frozen immediately at $-70^{\circ} \mathrm{C}$ until it was thawed for ELR ${ }^{+}$CXC chemokine ELISAs $(19,20,44,45)$. Throughout the entire study, we excluded any BALF obtained at a time when infection and/or colonization was diagnosed with the following criteria: a positive BALF or transbronchial biopsy by cytology or microbiology Gram stain and culture for bacterial, acid fast bacillus, fungus, cytomegalovirus (virology testing for CMV) included cytology, rapid shell-vial culture, and conventional tube culture), other respiratory virus, or Pneumocystis carinii pneumonia.

Diagnosis of BOS and healthy lung transplant recipients. Patients were diagnosed with BOS on the basis of an unexplained and sustained decrease in the forced expiratory volume in 1 second $\left(\mathrm{FEV}_{1}\right)$ by $20 \%$ or more of the peak predicted value after transplantation with or without pathologic evidence of BOS, as previously described (46). Patients diagnosed with acute rejection based on pathological findings by transbronchial biopsy were excluded (46). Healthy transplant recipients were those patients undergoing surveillance bronchoscopy for whom there had never been any clinical or biopsy evidence of acute rejection or BOS.
Immunosuppression and prophylactic antimicrobials. Patients were placed on a standard pre- and post-transplantation immunosuppression protocol including treatment with cyclosporine or tacrolimus, azathioprine or mycophenolate mofetil, methylprednisolone, and prophylactic antibiotics and antivirals, as previously described $(19,45)$. All episodes of acute lung allograft rejection were treated with a 3-day pulse of methylprednisolone at $1 \mathrm{~g} / \mathrm{d}$ without adjustment of daily prednisone.

Reagents. Abs to human CXCL1, CXCL3, CXCL5, CXCL7, CXCL8, VEGF, and murine VEGF were purchased from R\&D Systems. Biotinylated and non-biotinylated anti-murine CXCL1 was purchased from R\&D Systems. Polyclonal rabbit anti-murine CXCL2/3 Abs used for ELISAs were produced by the immunization of a rabbit with murine recombinant CXCL2/3 (R\&D Systems), as previously described (11, 47-50). Goat polyclonal $\mathrm{Ab}$ to murine and rabbit polyclonal $\mathrm{Ab}$ to human CXCR2 were generated using a 17 -mer peptide constituting the amino terminus of the mouse CXCR2 (MGEFKVDKFNIEDFFSG) and a 21-mer peptide constituting the amino terminus of human CXCR2 (CMEDFNMESDSFEDFWKGEDL), respectively, as previously described $(8,44,47,48)$. The anti-mouse CXCR2 Ab has been shown to be specific to rodent CXCR2 and does not alter peripheral blood neutrophil counts, as previously described $(10,11,18,47,48)$. The specificity of the anti-human CXCR2 $\mathrm{Ab}$ has been previously described (18). RB6-8C5 Ab is a rat anti-mouse monoclonal immunoglobulin $2 \mathrm{~b}$ against $\mathrm{Ly}-6 \mathrm{G}$, an antigen expressed on mature murine granulocytes but not their precursors, and has previously been shown to induce a neutrophil deficiency (51-55).

ELR ${ }^{+}$CXC chemokine ELISAs. We quantitated human CXCL1, CXCL3, CXCL5, CXCL7, CXCL8, and murine CXCL1 and CXCL2/3 protein using the double ligand method as previously described $(8,44)$.

Endothelial cell chemotaxis. HLMVECs at passage 5 were harvested by trypsinization and preincubated with either anti-hCXCR2 or control Ab at $37^{\circ} \mathrm{C}$ for 1 hour (Cambrex Corp.). Endothelial cell chemotaxis assays to a vehicle control, $50 \mathrm{ng} / \mathrm{ml}$ of CXCL8 and BALF pooled from 8 randomly selected patients from the FBOS, BOS, TBOS, and healthy groups were performed as previously described $(18,32)$. Results were expressed as the number of endothelial cells that migrated per high-power field (HPF).

CMP assay of angiogenesis. Angiogenic activity of BALF was assayed in vivo in the avascular cornea of hooded Long-Evans rat eyes, as previously described $(8,10,35)$. Human VEGF 165 and murine VEGF 164 (R\&D Systems) were used as positive controls when appropriate.

Murine BOS model. We used a well-established and reproducible murine model of BOS involving heterotopic subcutaneous trachea transplantation as previously described $(19,20)$. We used an MHC class I- and II-disparate combination of BALB/c $\left(\mathrm{H}-2^{\mathrm{d}}\right)$ to $\mathrm{C} 57 \mathrm{BL} / 6\left(\mathrm{H}-2^{\mathrm{b}}\right)$ (allografts) and $\mathrm{C} 57 \mathrm{BL} / 6$ $\left(\mathrm{H}-2^{\mathrm{b}}\right)$ to $\mathrm{C} 57 \mathrm{BL} / 6\left(\mathrm{H}-2^{\mathrm{b}}\right)$ (syngeneic controls). In separate experiments, animals received either anti-CXCR2 Ab or RB6-8C5 Ab or the combination of both as compared with appropriate control Abs on days 3, 5, 7, 9, 11, 13, and 15 by i.p. injection. In addition, to create experiments analogous to the clinical setting, we titrated the lowest dose of CsA (mixed in Cremophor EL castor oil; Sigma-Aldrich) given i.p. $(5,10,15,20$, and $25 \mathrm{mg} / \mathrm{kg} / \mathrm{d})$ to attenuate BOS at day 7, but not day 14, as compared to control (Cremophor EL). After determining that a dose of $20 \mathrm{mg} / \mathrm{kg} / \mathrm{d}$ significantly attenuated BOS at day 7 , but not day 14 , we then performed experiments in which CsA (20 $\mathrm{mg} / \mathrm{kg} / \mathrm{d}$ ) was injected i.p. into CXCR2 $2^{-/-}$and CXCR2 $2^{+/+}$allograft recipients on a C57/BL6 background starting at postoperative day 0 and given every day until sacrifice. All studies were approved by the UCLA institutional animal care and use committee.

Histopathologic grading of BOS. Three random $3-\mu \mathrm{m}$ paraffin-embedded tissue sections for 5 different trachea allografts were stained with $\mathrm{H} \& \mathrm{E}$ at 3 time points: days 7, 14, and 21. A blind assessment of the histopathology was performed using a modified histologic scoring system based on air- 
way lining epithelial loss, deposition of ECM, leukocyte infiltration, and luminal obliteration, as previously described $(19,20)$.

FACS analysis of neutrophils and endothelial cells. Tracheal single-cell suspension preparations were made using a previously described method $(19,44)$. Single-cell suspensions were stained with primary Abs: PerCP anti-mCD45 (BD Biosciences), PE anti-murine Ly-6G (neutrophil surface marker; BD Biosciences), biotin-conjugated anti-mouse MECA-32 (BD Biosciences), and anti-CXCR2 or isotype controls. The secondary Abs used for these experiments was Alexa Fluor 488 (Invitrogen Corp.) for CXCR2 and streptavidinPE (BD Biosciences) for MECA-32. Dual-color-stained cell suspensions were analyzed on a FACScan flow cytometer using CellQuest 3.2.1f1 software (BD Biosciences - Immunocytometry Systems) as previously described $(19,44)$.

Total RNA isolation and real-time quantitative PCR. Total cellular RNA was isolated as previously described $(8,44)$. Total RNA was determined and reversed transcribed into cDNA and amplified using TaqMan Reverse Transcription Reagents (Applied Biosystems). We performed real-time quantitative PCR with specific TaqMan primers and probes, using the ABI Prism 7700 Sequence Detector and SDS analysis software (Applied Biosystems). Negative control experiments were performed as follows: realtime quantitative PCR was performed without RT to exclude contamination and amplification of genomic DNA; and without cDNA template to exclude reagent contamination with DNA. CXCL1, CXCR2, and 18s TaqMan Pre-Developed Assay Reagent (PDAR) (Applied Biosystems) were used, and CXCL2/3 primers and probe sequence (forward primer, reverse primer, TaqMan probe) were as follows: AGGGCAGGAGCGAATTTAAAA, TGCGATCTCCCCACAGCT, AGGCCAGGGTGGGCGGAGG.

Quantitative analysis of gene expression was done using the comparative $C_{\mathrm{T}}\left(\Delta C_{\mathrm{T}}\right)$ method, in which $C_{\mathrm{T}}$ is the threshold cycle number $(20,56)$. The formula for the $\Delta \mathrm{C}_{\mathrm{T}}$ method is described as the difference in threshold cycles for a target (i.e., CXCL1) and an endogenous reference (i.e., housekeeping gene 18S). The amount of target normalized to an endogenous reference (i.e., CXCL1 in allografts at day 3 ) and relative to a calibration normalized to an endogenous reference (i.e., CXCL1 in syngeneic controls at day 3 ) is given by $2^{-\Delta \triangle C T}$, which is the fold increase in CXCL1 of the allograft compared with the syngeneic control, as previously described $(20,56)$.

Immunohistochemistry for F8Ag and CXCR2. Three random 3- $\mu \mathrm{m}$ paraffin-embedded tissue sections from 5 human BOS patients compared with normal lung tissue controls were assessed by immunohistochemical localization of either appropriate control Ab, F8Ag, or CXCR2 using goat antiF8Ag (Biomeda Corp.) and rabbit anti-hCXCR2 as previously described (10). Three random $3-\mu \mathrm{m}$ paraffin-embedded tissue sections from 5 different day 7 and day 21 murine tracheal allografts, compared with 5 different day 7 and day 21 syngeneic control tissues, were processed for immunohistochemical localization of either appropriate control Ab, goat anti-F8Ag (Biomeda Corp.), or goat anti-mCXCR2 as previously described (10). Morphometric analysis of vascular remodeling was quantitated by counting the number of F8Ag-positive vessels per $\times 400 \mathrm{HPF}(n=50 ; 5$ measurements from 2 sections of each of the 5 sample blocks).

Hydroxyproline assay. Prior to removal, heterotopically transplanted tracheas were dissected free of surrounding tissue, and total tracheal collagen was determined by analysis of hydroxyproline as previously described $(8,19,20)$.

Statistical analysis. Data were analyzed using the Statview 4.5 statistical software package (Abacus Concepts Inc.). All human group comparisons were evaluated by the nonparametric Kruskal-Wallis test with Dunn posthoc analysis. Data are displayed using a box plot summary. The horizontal line represents the median, the box encompasses the 25 th to 75 th percentile, and the error bars encompass the 10th to the 90th percentile. All animal group comparisons were evaluated by the ANOVA test with Bonferroni/Dunn post-hoc analysis. Data are expressed as mean \pm SEM.

\section{Acknowledgments}

This work was supported, in part, by grants from the NIH (HL04493 and HL080206-01 to John A. Belperio; P01HL67665, CA87879, P50CA90388, and HL66027 to Robert M. Strieter).

Received for publication December 17, 2004, and accepted in revised form February 22, 2005.

Address correspondence to: John A. Belperio, Department of Medicine, Division of Pulmonary and Critical Care Medicine, David Geffen School of Medicine at University of California, Los Angeles, Room 14-154 Warren Hall, Box 711922, 900 Veteran Avenue, Los Angeles, California 90095-1786, USA. Phone: (310) 794-1999; Fax: (310) 794-1998; E-mail: jbelperio@mednet.ucla.edu.
1. Trulock, E.P. 1997. Lung transplantation. Am. J. Respir. Crit. Care Med. 155:789-818.

2. Arcasoy, S.M., and Kotloff, R.M. 1999. Lung transplantation. N. Engl. J. Med. 340:1081-1091.

3. Folkman, J. 1995. Angiogenesis in cancer, vascular, rheumatoid and other disease. Nat. Med. 1:27-31.

4. Belperio, J.A., et al. 2000. CXC chemokines in angiogenesis. J. Leukoc. Biol. 68:1-8.

5. Strieter, R.M., Belperio, J.A., and Keane, M.P. 2003. $\mathrm{CXC}$ chemokines in vascular remodeling related to pulmonary fibrosis. Am. J. Respir. Cell Mol. Biol. 29(Suppl. 3):S67-S69.

6. Sivakumar, B., Harry, L.E., and Paleolog, E.M. 2004 Modulating angiogenesis: more vs. less. JAMA. 292:972-977.

7. Koch, A.E. 2003. Angiogenesis as a target in rheumatoid arthritis. Ann. Rheum. Dis. 62(Suppl. 2):ii60-ii67.

8. Keane, M.P., et al. 1999. Neutralization of the CXC chemokine, macrophage inflammatory protein-2, attenuates bleomycin-induced pulmonary fibrosis. J. Immunol. 162:5511-5518.

9. Garkavtsev, I., et al. 2004. The candidate tumour suppressor protein ING4 regulates brain tumour growth and angiogenesis. Nature. 428:328-332.

10. Keane, M.P., et al. 2002. Imbalance in the expression of CXC chemokines correlates with bronchoalveolar lavage fluid angiogenic activity and procollagen levels in acute respiratory distress syndrome. J. Immunol. 169:6515-6521.
11. Keane, M.P., Belperio, J.A., Xue, Y.Y., Burdick, M.D., and Strieter, R.M. 2004. Depletion of CXCR2 inhibits tumor growth and angiogenesis in a murine model of lung cancer. J. Immunol. 172:2853-2860.

12. Slebos, D.J., et al. 2004. Bronchoalveolar lavage fluid characteristics in acute and chronic lung transplant rejection. J. Heart Lung Transplant. 23:532-540.

13. Elssner, A., et al. 2000. Elevated levels of interleukin-8 and transforming growth factor-beta in bronchoalveolar lavage fluid from patients with bronchiolitis obliterans syndrome: proinflammatory role of bronchial epithelial cells. Munich Lung Transplant Group. Transplantation. 70:362-367.

14. Riise, G.C., et al. 1999. Persistent high BAL fluid granulocyte activation marker levels as early indicators of bronchiolitis obliterans after lung transplant. Eur. Respir. J. 14:1123-1130.

15. DiGiovine, B., et al. 1996. Bronchoalveolar lavage neutrophilia is associated with obliterative bronchiolitis after lung transplantation: role of IL-8. J. Immunol. 157:4194-4202.

16. Strieter, R.M., Belperio, J.A., Phillips, R.J., and Keane, M.P. 2004. CXC chemokines in angiogenesis of cancer. Semin. Cancer Biol. 14:195-200.

17. Strieter, R.M., Belperio, J.A., Phillips, R.J., and Keane, M.P. 2004. Chemokines: angiogenesis and metastases in lung cancer. Novartis Found. Symp.
256:173-184; discussion 184-188, 259-269.

18. Addison, C.L., et al. 2000. The CXC chemokine receptor 2, CXCR2, is the putative receptor for ELR+ CXC chemokine-induced angiogenic activity. J. Immunol. 165:5269-5277.

19. Belperio, J.A., et al. 2001. Critical role for the chemokine MCP-1/CCR2 in the pathogenesis of bronchiolitis obliterans syndrome. J. Clin. Invest. 108:547-556. doi:10.1172/JCI200112214.

20. Belperio, J.A., et al. 2002. Critical role for CXCR3 chemokine biology in the pathogenesis of Bronchiolitis obliterans syndrome. J. Immunol. 169:1037-1049.

21. Aalamian, Z. 2001. Reducing adverse effects of immunosuppressive agents in kidney transplant recipients. Prog. Transplant. 11:271-282; quiz 283-284.

22. Riise, G.C., et al. 1998. Bronchiolitis obliterans syndrome in lung transplant recipients is associated with increased neutrophil activity and decreased antioxidant status in the lung. Eur. Respir. J. 12:82-88.

23. Zheng, L., et al. 2000. Airway neutrophilia in stable and bronchiolitis obliterans syndrome patients following lung transplantation. Thorax. 55:53-59.

24. Elssner, A., and Vogelmeier, C. 2001. The role of neutrophils in the pathogenesis of obliterative bronchiolitis after lung transplantation. Transpl. Infect. Dis. 3:168-176.

25. Hopkins, P.M., et al. 2004. Association of minimal rejection in lung transplant recipients with oblit- 
erative bronchiolitis. Am. J. Respir. Crit. Care Med. 170:1022-1026.

26. Burger, M., et al. 1999. Point mutation causing constitutive signaling of CXCR2 leads to transforming activity similar to Kaposi's sarcoma herpesvirus-G protein-coupled receptor. J. Immunol. 163:2017-2022.

27. Richards, B.L., Eisma, R.J., Spiro, J.D., Lindquist, R.L., and Kreutzer, D.L. 1997. Coexpression of interleukin-8 receptors in head and neck squamous cell carcinoma. Am. J. Surg. 174:507-512.

28. Nanney, L.B., Mueller, S.G., Bueno, R., Peiper, S.C., and Richmond, A. 1995. Distributions of melanoma growth stimulatory activity of growth-regulated gene and the interleukin- 8 receptor $B$ in human wound repair. Am. J. Pathol. 147:1248-1260.

29. Lee, J., et al. 1992. Characterization of two high affinity human interleukin-8 receptors. J. Biol. Chem. 267:16283-16287.

30. Ahuja, S.K., and Murphy, P.M. 1996. The CXC chemokines growth-regulated oncogene (GRO) alpha, GRObeta, GROgamma, neutrophil-activating peptide-2, and epithelial cell-derived neutrophil-activating peptide-78 are potent agonists for the type B, but not the type A, human interleukin- 8 receptor. J. Biol. Chem. 271:20545-20550.

31. Schonbeck, U., Brandt, E., Petersen, F., Flad, H.D., and Loppnow, H. 1995. IL-8 specifically binds to endothelial but not to smooth muscle cells. J. Immunol. 154:2375-2383.

32. Strieter, R.M., et al. 1995. The functional role of the ELR motif in CXC chemokine-mediated angiogenesis. J. Biol. Chem. 270:27348-27357.

33. Luan, J., et al. 1997. Mechanism and biological significance of constitutive expression of MGSA/ GRO chemokines in malignant melanoma tumor progression. J. Leukoc. Biol. 62:588-597.

34. Miller, L.J., et al. 1998. Expression of interleukin-8 receptors on tumor cells and vascular endothelial cells in human breast cancer tissue. Anticancer Res. 18:77-81.
35. Keane, M.P., et al. 2001. ENA-78 is an important angiogenic factor in idiopathic pulmonary fibrosis. Am. J. Respir. Crit. Care Med. 164:2239-2242.

36. Meyer, K.C., Cardoni, A.L., Xiang, Z., Cornwell, R.D., and Love, R.B. 2001. Vascular endothelial growth factor in human lung transplantation. Chest. 119:137-143.

37. Genden, E.M., Iskander, A., Bromberg, J.S., and Mayer, L. 2003. The kinetics and pattern of tracheal allograft re-epithelialization. Am. J. Respir. Cell Mol. Biol. 28:673-681.

38. Mitzner, W., and Wagner, E.M. 2004. Vascular remodeling in the circulations of the lung. J. Appl. Physiol. 97:1999-2004.

39. Scapini, P., et al. 2004. CXCL1/macrophage inflammatory protein-2-induced angiogenesis in vivo is mediated by neutrophil-derived vascular endothelial growth factor-A. J. Immunol. 172:5034-5040.

40. Benelli, R., et al. 2002. Neutrophils as a key cellular target for angiostatin: implications for regulation of angiogenesis and inflammation. FASEB J. 16:267-269.

41. Eppinger, M.J., Jones, M.L., Deeb, G.M., Bolling, S.F., and Ward, P.A. 1995. Pattern of injury and the role of neutrophils in reperfusion injury of rat lung. J. Surg. Res. 58:713-718.

42. Eppinger, M.J., Deeb, G.M., Bolling, S.F., and Ward, P.A. 1997. Mediators of ischemia-reperfusion injury of rat lung. Am. J. Pathol. 150:1773-1784.

43. Morita, K., et al. 2001. Early chemokine cascades in murine cardiac grafts regulate $T$ cell recruitment and progression of acute allograft rejection. J. Immunol. 167:2979-2984.

44. Belperio, J.A., et al. 2000. The role of the CC chemokine, RANTES, in acute lung allograft rejection. J. Immunol. 165:461-472.

45. Belperio, J.A., et al. 2002. Interleukin-1 receptor antagonist as a biomarker for bronchiolitis obliterans syndrome in lung transplant recipients. Transplantation. 73:591-599.

46. Yousem, S.A., et al. 1996. Revision of the 1990 working formulation for the classification of pulmonary allograft rejection: Lung Rejection Study Group. J. Heart Lung Transplant. 15:1-15.

47. Mehrad, B., et al. 1999. CXC chemokine receptor-2 ligands are necessary components of neutrophilmediated host defense in invasive pulmonary aspergillosis. J. Immunol. 163:6086-6094.

48. Moore, T.A., et al. 2000. Bacterial clearance and survival are dependent on CXC chemokine receptor-2 ligands in a murine model of pulmonary Nocardia asteroides infection. J. Immunol. 164:908-915.

49. Sue, R.D., et al. 2004. CXCR2 is critical to hyperoxiainduced lung injury. J. Immunol. 172:3860-3868.

50. Belperio, J.A., et al. 2002. Critical role for CXCR2 and CXCR2 ligands during the pathogenesis of ventilator-induced lung injury. J. Clin. Invest. 110:1703-1716. doi:10.1172/JCI200215849.

51. Mehrad, B., et al. 2002. Transient lung-specific expression of the chemokine $\mathrm{KC}$ improves outcome in invasive aspergillosis. Am. J. Respir. Crit. Care Med. 166:1263-1268.

52. Mehrad, B., Strieter, R.M., and Standiford, T.J. 1999. Role of TNF-alpha in pulmonary host defense in murine invasive aspergillosis. J. Immunol. 162:1633-1640

53. Fleming, T.J., Fleming, M.L., and Malek, T.R. 1993. Selective expression of Ly-6G on myeloid lineage cells in mouse bone marrow. RB6-8C5 mAb to granulocyte-differentiation antigen (Gr-1) detects members of the Ly- 6 family. J. Immunol. 151:2399-2408.

54. Tateda, K., et al. 2001. Early recruitment of neutrophils determines subsequent $\mathrm{T} 1 / \mathrm{T} 2$ host responses in a murine model of Legionella pneumophila pneumonia. J. Immunol. 166:3355-3361.

55. Schon, M., Denzer, D., Kubitza, R.C., Ruzicka, T., and Schon, M.P. 2000. Critical role of neutrophils for the generation of psoriasiform skin lesions in flaky skin mice. J. Invest. Dermatol. 114:976-983.

56. Belperio, J.A., et al. 2003. Role of CXCL9/CXCR3 chemokine biology during pathogenesis of acute lung allograft rejection. J. Immunol. 171:4844-4852. 